\title{
Variabilidad estacional de cadmio en un sistema de surgencia costera del norte de Chile (Bahía Mejillones del Sur, 23 $\mathrm{S}$ )
}

\author{
Seasonal variability of cadmium in a coastal upwelling system off northern Chile \\ (Mejillones bay, $23^{\circ} \mathrm{S}$ )
}

\author{
JORGE VALDÉS ${ }^{1}$, DOMINGO ROMÁN ${ }^{2}$, PAOLA DÁVILA ${ }^{3}$ LUC ORTLIEB $^{4} \&$ MARCOS GUÍNẼEZ $^{1,5}$
}

\begin{abstract}
${ }^{1}$ Laboratorio de Sedimentología y Paleoambientes, Instituto de Investigaciones Oceanológicas, Facultad de Recursos del Mar, Universidad de Antofagasta, Casilla 170, Antofagasta, Chile; jvaldes@uantof.cl

${ }^{2}$ Departamento de Química, Facultad de Ciencias Básicas, Universidad de Antofagasta, Chile; droman@uantof.cl

${ }^{3}$ Instituto de Investigaciones Oceanológicas, Facultad de Recursos del Mar, Universidad de Antofagasta, Casilla 170, Antofagasta, Chile; pdavila@uantof.cl

${ }^{4}$ UR 055, Paleotropique, Institut de Recherche pour le Developpement, Francia; Luc.Ortlieb@ bondy.ird.fr

${ }^{5}$ Programa de Doctorado en Ciencias Aplicadas, Mención Sistemas Marinos Costeros, Facultad de Recursos del Mar, Universidad de Antofagasta; mguinez@uantof.cl
\end{abstract}

\begin{abstract}
RESUMEN
Entre junio de 2002 y abril de 2003 se midió el contenido de cadmio total y disuelto entre la superficie y los $100 \mathrm{~m}$ de profundidad, en dos estaciones localizadas en la bahía Mejillones del Sur. El rango de cadmio total y cadmio disuelto en las aguas de esta bahía fue 0,41-10,7 y 0,08-1,61 nM, respectivamente, durante el período de muestreo. Los resultados fueron correlacionados con la estructura físico-química de la columna de agua al momento del muestreo, de manera de estudiar los patrones de variabilidad estacional de este metal en este sistema de surgencia. Las condiciones oceanográficas imperantes durante este período correspondieron a un año normal (no-El Niño). Las masas de agua presentes en la bahía correspondieron a agua subantártica, subtropical y ecuatorial subsuperficial, con diferentes grados de mezcla y estratificación durante el período de estudio. Los perfiles de cadmio presentaron una distribución vertical clásica de un micronutriente. Sin embargo estos perfiles presentaron leves diferencias que pueden ser atribuidas a la condición de mezcla de las masas de agua al interior de la bahía. Un análisis multivariado de los parámetros registrados indicó que la variación temporal de cadmio en las aguas superficiales está asociada a las fluctuaciones de la clorofila $a$ y la temperatura. Esto sugiere que, a escala estacional, la producción de biomasa fitoplanctónica sería el principal factor que controla la distribución de cadmio en esta bahía. Por otro lado, a pesar de que el cadmio es un metal redox sensitivo no se encontró un efecto significativo de la disponibilidad de oxígeno sobre el comportamiento de este metal, de manera que la condición micróxica de las aguas de fondo de la bahía no sería un factor preponderante en el flujo de cadmio hacia los sedimentos. La razón $\mathrm{Cd} / \mathrm{PO}_{4}$ encontrada en Mejillones es similar a la reportada en otras zonas costeras de Chile. Los bajos valores y la tendencia temporal observada en esta razón pueden ser explicados ya sea por una mayor eficiencia en la remoción de $\mathrm{PO}_{4}$ que de Cadmio en las aguas superficiales o una mayor remineralización de cadmio en las aguas subsuperficiales.
\end{abstract}

Palabras clave: cadmio, sistema de surgencia, Mejillones, Chile.

\section{ABSTRACT}

Total and dissolved cadmium were measured from surface to $100 \mathrm{~m}$ water depth, between June 2002 and April 2003, in Mejillones bay. Total and dissolved cadmium range concentration was 0.41-10.7 and 0.08-1.61 $\mathrm{nM}$, respectively. Cadmium profiles and water structure were used to study seasonal variability patterns of this metal in this upwelling system. Oceanographic conditions during this period correspond to a normal (nonEl Niño) year. Water masses present in this bay correspond to subantartic superficial water, subtropical superficial water and Equatorial subsuperficial water with different degree of mixture and stratification during sampling period. Cadmium profiles showed a classic nutrient-type distribution. Whereas some differences in this profiles could be attributed to a water masses mixing condition into the bay. Multivariate analysis indicates that temporal cadmium variations were associated with chlorophyll-a concentration and temperature fluctuation, indicating that, at this temporal resolution, phytoplanktonic biomass production is the principal factor controlling cadmium concentration in surface waters of Mejillones bay. By other hand, in spite of cadmium being a redox-sensitive metal, any significant influence of dissolved oxygen on this metal were founded, thus microxic condition of bottom water of Mejillones bay is not the principal factor that promotes 
cadmium flux to the sediments. $\mathrm{Cd} / \mathrm{PO}_{4}$ ratio is in agreement with normal values reported for the Chilean coast, and its low values, compared with the northeast Pacific coast, can be explained either by the fact that $\mathrm{PO}_{4}$ is more efficiently removed than cadmium from the surface layer, or that cadmium remineralization is occurring much faster than in the case of $\mathrm{PO}_{4}$.

Key words: cadmium, upwelling system, Mejillones, Chile.

\section{INTRODUCCIÓN}

En las últimas décadas ha aumentado considerablemente nuestro conocimiento sobre la biogeoquímica de los metales traza en el ambiente marino. Los estudios se han enfocado en realizar inventarios de metales en aguas costeras y oceánicas y en describir, para luego intentar explicar, el rol que juegan estos metales en los procesos biológicos que tienen lugar en el océano (Bruland 1980, Klinkhammer \& Bender 1980, Kremling 1985, Saager et al. 1992, Cotté-Krief et al. 2000). Uno de los metales que más atención ha generado en el último tiempo es el cadmio (Cd), el cual presenta solo un estado de oxidación en agua de mar ( Cd II), encontrándose como $\mathrm{CdCl}^{+}$(Morford \& Emerson 1999, Russell \& Morford 2001, Nameroff et al. 2002). Este metal se encuentra en mayores concentraciones en las aguas costeras que en las aguas oceánicas (Kremling 1985, Muller et al. 1994, Le Gall et al. 1999), lo cual es el resultado de la interacción océanocontinente, en donde los mecanismos de transporte continentales (ríos, viento) y los propiamente marinos (surgencia) afectan la composición geoquímica de las aguas costeras (Cotté-Krief et al. 2000). Particular interés ha despertado la asociación existente entre las distribuciones de Cadmio y algunos nutrientes como nitrato y fosfato en la columna de agua (Elbaz-Poulichet et al. 1987, Abe \& Matsunaga 1988, Bruland et al. 1994, de Baar et al. 1994, Abe 2001). En términos simples, este metal es removido de la capa superficial del océano mediante incorporación en el tejido biológico de organismos planctónicos, y posteriormente, durante el proceso de sedimentación del material biogénico la oxidación de la materia orgánica devuelve los nutrientes y el $\mathrm{Cd}$ al medio acuático, los cuales son nuevamente transportados hacia la superficie por los fenómenos de surgencia (Bruland 1980).

La variación temporal del contenido de $\mathrm{Cd}$ de las aguas superficiales ha sido estudiada en diversos sistemas costeros en relación a la intensidad de la surgencia, eventos El Niño y la productividad biológica (Boyle 1988, Van Geen et al. 1992, Van Geen \& Husby 1996, Takesue et al. 2004). Diversos mecanismos han sido propuestos para explicar la geoquímica de este metal en el ambiente marino. Por una parte, se ha reportado que el oxígeno disuelto juega un rol preponderante en el comportamiento de este metal en las aguas subsuperficiales ya que tiende a precipitar cuando se generan condiciones microxicas/ anóxicas próximas al piso marino (Saager et al. 1992, Rosenthal et al. 1995, van Geen et al. 1995, Mosford \& Emerson 1999). Por otro lado, se ha propuesto que la surgencia es uno de los mecanismos más importantes que regulan la distribución de $\mathrm{Cd}$ en las aguas superficiales de diferentes ambientes costeros (Van Geen et al. 1992, Van Geen \& Husby 1996). Ambas características han fundamentado su utilización como un marcador de circulación de masas de agua y de reconstrucciones paleoceanográficas (Boyle 1988, Takesue et al. 2004). Sin embargo, no es posible generalizar estas interpretaciones a todos los ambientes costeros, por lo que se hace necesario evaluar dichas hipótesis en cada uno de los ecosistemas que presentan características oceanográficas propicias (intensa surgencia, bajos niveles de oxígeno en el agua subsuperfical) para realizar reconstrucciones paleoambientales (Nameroffet al. 2002, Takesue et al. 2004).

\section{Oceanografía local}

La corriente de Humboldt es uno de los sistemas más productivos de mundo. Los altos niveles de captura de peces pelágicos, especialmente en la zona norte de este sistema, son explicados por la existencia de centros de surgencia que soportan toda la productividad biológica de la zona (Strub et al. 1998). Uno de estos centros de surgencia está ubicado en Punta Angamos, al noroeste de la bahía Mejillones del Sur (Fig. 1). 


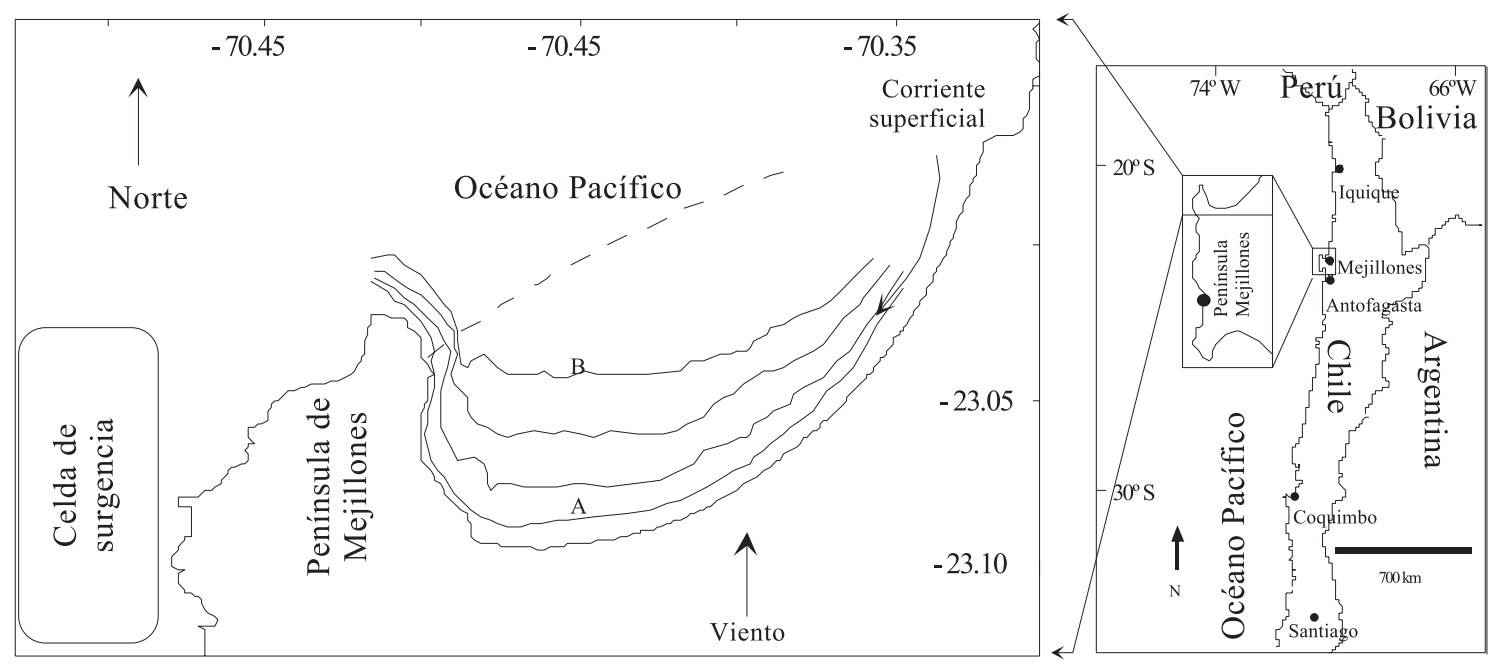

Fig.1: Localización de los puntos de muestreo de agua superficial en la bahía Mejillones del Sur; (A) corresponde a estación costera, (B) a estación pelágica. Se muestra las principales características oceanográficas de la zona. La línea discontinua indica la posición del frente térmico que genera una "sombra de surgencia" en la bahía, según Marín et al. (2003). El círculo negro mostrado en la Península de Mejillones corresponde la localidad de muestreo de Isla Santa María, reportado en el trabajo de Takesue et al. (2004).

Location of sampling stations in Mejillones bay; (A) is the coastal station, (B) is the pelagic station. Principal oceanographic characteristics of this zone are showed. The dashed line corresponds to a thermal front responsible for an "upwelling shadow" in Mejillones bay, according to Marin et al. (2003). Filled circle shown in Mejillones Peninsula corresponds to Santa María location, reported by Takesue et al. (2004).

En esta zona se han realizado numerosos estudios relacionados con las características biológicas y físicas de este sistema, generando importante información, especialmente, respecto de su respuesta a eventos océanoclimáticos como El Niño (Escribano 1998, González et al. 1998, 2000, 2004, Iriarte et al. 2000, 2004, Sobarzo \& Figueroa 2001, Ulloa et al. 2001, Pizarro et al. 2002).

La bahía Mejillones presenta dos características oceanológicas principales; valores de productividad primaria de $1.070 \mathrm{~g}$ C m² año-1 (Rodríguez et al. 1986, 1991, Marín et al. 1993) y una Zona de Mínimo Oxígeno (ZMO; oxígeno disuelto $<0,5 \mathrm{~mL}$ $\left.\mathrm{L}^{-1}\right)$ que se ubica a partir de aproximadamente los 50-60 m de profundidad, dependiendo de la época del año (Marín et al. 1993, Escribano 1998, González et al. 1998, 2000, Valdés 1998, Marín \& Olivares 1999, Escribano \& Hidalgo 2000). La bahía Mejillones presenta condiciones que favorecen el incremento de la productividad biológica superficial, debido a la existencia de un frente térmico que le confiere una mayor estabilidad hidrodinámica y una permanencia más prolongada de los nutrientes en la capa fótica, característica denominada "sombra de surgencia" (Marín \& Olivares 1999, Marín et al. 2003). Por otra parte, la extrema aridez de esta región provoca que el aporte continental a la sedimentación marina está restringido al transporte eólico de partículas litogénicas (Vargas 2002), y por ello, la surgencia parece ser uno de los procesos más importantes que explicarían las características físico-química de las aguas de la bahía. Estas características ofrecen una excelente oportunidad para estudiar la influencia de los procesos biológicos sobre la geoquímica de los metales traza presentes en el ambiente marino. Sin embargo, a la fecha, solamente existen trabajos relacionados con la distribución de diversos metales, entre ellos el $\mathrm{Cd}$, en los sedimentos de fondo, tanto para evaluar eventuales problemas de contaminación (Valdés et al. 2005), como para reconstruir variaciones en las condiciones de oxigenación de fondo durante el pasado reciente (Valdés \& Ortlieb 2001, Valdés et al. 2003, 2004). 
Este trabajo entrega las primeras mediciones de la concentración de $\mathrm{Cd}$, durante un ciclo anual, en la bahía Mejillones del Sur, parte integrante del centro de surgencia de Punta Angamos. El objetivo de este estudio es describir los patrones estacionales de variabilidad de Cd en esta bahía. Se utilizaron dos enfoques para evaluar este comportamiento; uno de ellos está relacionado con la distribución de $\mathrm{Cd}$ de las aguas superficiales (0-5 $\mathrm{m}$ de profundidad) y el otro corresponde a su distribución en la columna de agua entre 0 y $100 \mathrm{~m}$ de profundidad. Con esto se pretende identificar los factores responsables de la variación superficial de $\mathrm{Cd}$, eventuales diferencias en su distribución espacial, evaluar la influencia del oxígeno disuelto sobre la distribución vertical de Cd, y la relación de este metal con las masas de agua presentes al interior de la bahía Mejillones.

\section{MATERIALES Y MÉTODOS}

\section{Procedimiento de muestreo}

Entre junio de 2002 y abril de 2003 se recolectaron, bimensualmente, muestras de agua en dos puntos de la bahía Mejillones del Sur (Fig. 1). El muestreo se realizó cada $5 \mathrm{~m}$ de profundidad, entre la superficie y los $30 \mathrm{~m}$ en la estación costera (Estación A), y hasta los $100 \mathrm{~m}$ en la estación pelágica (Estación B). Para ello se utilizó una botella oceanográfica Niskin (General Oceanic) de $5 \mathrm{~L}$, y un cable oceanográfico de acero inoxidable. Todas las muestras fueron almacenadas a baja temperatura, en botellas de polietileno de alta densidad. Tanto la botella de muestreo como las de almacenamiento fueron tratadas con ácido nítrico y ácido clorhídrico de acuerdo a la metodología propuesta por Kremling (1983). Antes de cada muestreo las botellas fueron lavadas tres veces con la misma agua que se estaba colectando. Los parámetros oceanográficos (temperatura, salinidad y oxígeno disuelto) fueron medidos con un equipo CTD, marca SeaBird 19 plus, en las mismas estaciones de muestreo.

\section{Procedimiento analítico: metales traza}

La concentración de $\mathrm{Cd}$ total $\left(\mathrm{Cd}_{\text {total }}\right)$ y de $\mathrm{Cd}$ disuelto $\left(\mathrm{Cd}_{\mathrm{dis}}\right)$ fue medida por la técnica de análisis potenciométrico (Estela et al. 1995). Para ello se utilizó un equipo prototipo acoplado a una estación de muestras SAM 20 (Radiometer, Copenhagen, Denmark) y conectado a un computador IBM compatible. Este equipo es operado bajo control de un programa computacional dedicado (plataforma Windows) y de esta forma el procedimiento analítico puede ser completamente controlado. La celda electroanalítica la conforman un electrodo de trabajo de carbón vítreo pretratado (Bioanalytical Sistem MF 2012; Radiometer F-3500 y F-3600), un electrodo de referencia de doble cámara $\mathrm{AgCl} / \mathrm{Ag}$ (Orion 900200) y un electrodo auxiliar de alambre de $\mathrm{Pt}$ (Radiometer P-1312).

Para determinar la concentración disuelta, las muestras de agua fueron filtradas con membranas Millipore de $0,45 \mu \mathrm{m}$, y llevadas hasta $\mathrm{pH} 2$ con ácido nítrico. Este procedimiento se realizó inmediatamente después del muestreo, dentro de una campana de flujo laminar (Purifier Class II, Labconco, Kansas, USA). Posteriormente las muestras filtradas fueron mantenidas a $4{ }^{\circ} \mathrm{C}$ hasta el momento del análisis.

Para determinar la concentración total, las muestras de agua fueron llevadas hasta $\mathrm{pH} 2$ con ácido nítrico. Posteriormente se realizó una digestión de una submuestra de $250 \mathrm{~mL}$ con ácido nítrico, a una temperatura de $90{ }^{\circ} \mathrm{C}$, durante dos horas. La acidez final de la solución resultante fue de $1 \mathrm{M}$ en $\mathrm{HNO}_{3}$. El procedimiento analítico fue controlado mediante el análisis rutinario de réplicas, blancos y material de referencia certificado CASS-4 (coastal seawater) (Tabla 1).

\section{TABLA 1}

Validación analítica de la determinación de cadmio en agua de mar

Analytical validation of the determination of cadmium in seawater

\begin{tabular}{lc}
\hline Parámetro & Resultados \\
\hline Concentración medida $\left(\mu \mathrm{g} \mathrm{L}^{-1}\right)$ & 0,027 \\
Concentración certificada $\left(\mu \mathrm{g} \mathrm{L}^{-1}\right)$ & \\
CASS-4 (NRC-CNRC) & 0,026 \\
Desviación estándar $( \pm \%)$ & 2,5 \\
Error $(\%)$ & 3,8 \\
\hline
\end{tabular}


Procedimiento analítico: nutrientes y clorofila a

Estos análisis fueron realizados en muestras de agua superficial, provenientes de las dos estaciones de colecta. La concentración de nitrato y fosfato fue determinada en un espectrofotómetro (Spectronic 20D, New York, USA) siguiendo la metodología propuesta por Parsons et al. (1984), previa filtración en membranas de 0,45 $\mathrm{mm}$ de diámetro de poro. La concentración de clorofila $a$ fue media en un espectrofluorómetro RF-5301 (Shimadzu, Kyoto, Japón), de acuerdo a la metodología propuesta por Holm-Hansen et al. (1965). Los resultados de nutrientes son expresados en umol L $\mathrm{L}^{-1}$, y los resultados de clorofila son expresados en $\mu \mathrm{g} \mathrm{L} \mathrm{L}^{-1}$.

\section{RESULTADOS}

Los perfiles de temperatura, salinidad y oxígeno disuelto son mostrados en la Fig. 2. Se observó un buen acoplamiento de la termoclina, haloclina y oxiclina durante la época de muestreo, ubicándose más cerca de la superficie durante el período estival y profundizándose durante el periodo invernal. La variabilidad bimensual de la temperatura en la capa superficial, preferentemente hasta los $30 \mathrm{~m}$ de profundidad, presentó un patrón que puede ser asociado a la evolución de la radiación solar para esta latitud. Los valores máximos se registraron en diciembre, mientras que los mínimos lo hicieron en agosto. Por otra parte desde los $30 \mathrm{~m}$ en adelante la temperatura se mantuvo cercana al valor de $13{ }^{\circ} \mathrm{C}$ en todo el período de estudio.

Los perfiles de salinidad mostraron una mayor variabilidad en la capa comprendida entre 10 y $30 \mathrm{~m}$, excepto en agosto mes en el cual dicha capa se ubicó entre los $30 \mathrm{~m}$ y $70 \mathrm{~m}$ de profundidad. Los meses de junio y agosto presentan valores mínimos de salinidad comprendidos entre 34,5 a 34,7 , mientras que el mes de abril sobresale por un máximo de salinidad de 35,1 en la capa de 15 a $20 \mathrm{~m}$ de profundidad. A partir de los $70 \mathrm{~m}$ la salinidad converge a 34,8 , durante todo el período de estudio. Los perfiles de contenido de oxígeno disuelto presentaron una mayor variabilidad en superficie con valores mínimos en febrero y máximos en diciembre y abril. Se utilizó la escala de Bernhard \& Sen Gupta (1999) para diferenciar ambientes óxicos $\left(>1 \mathrm{~mL} \mathrm{~L}^{-1}\right)$, disóxico (entre $1 \mathrm{~mL} \mathrm{~L}^{-1}$ y $0,1 \mathrm{~mL} \mathrm{~L}^{-1}$ ), micróxico $\left(<0,1 \mathrm{~mL} \mathrm{~L}^{-1}\right.$ ), y anóxico (oxígeno no detectable), en la bahía Mejillones. A partir de los $30 \mathrm{~m}$ el contenido de oxígeno en la columna de agua se reduce considerablemente generando condiciones micróxicas durante todo el período de estudio, a excepción del mes de agosto, donde los valores menores a $0,1 \mathrm{~mL} \mathrm{~L}^{-1}$ se registraron a partir de $10 \mathrm{~s} 70 \mathrm{~m}$.

De acuerdo a la variación bimensual de la temperatura, salinidad y oxígeno registrados al interior de la bahía Mejillones, se observaron características de tres masas de agua comúnmente identificadas en la zona frente a la misma (Strub et al. 1998, Sobarzo \& Figueroa 2001). Dichas masas de agua corresponden al agua superficial subtropical (ASST), agua superficial subantártica (ASSA) y agua ecuatorial subsuperficial (AESS) (Fig. 3). Los meses de junio y agosto mostraron un predominio ASSA en la capa superficial y/o intermedia (Fig. 3), caracterizada por los bajos valores de salinidad y altos valores de contenido de oxígeno. El mes de octubre presentó características de mezcla de AESS con remanentes de ASSA (Fig. 3). Por debajo de los $20 \mathrm{~m}$ predominan las características hidrográficas asociadas al AESS. El mes de diciembre presentó características correspondientes a ASSA y ASST, en la capa superficial (Fig. 3). Por un lado, la baja salinidad $(34,6$ a $15 \mathrm{~m})$ y alto contenido de oxígeno en superficie del orden de $8 \mathrm{~mL} \mathrm{~L}^{-1}$, concuerda con características de ASSA. Sin embargo la elevada temperatura, de hecho la máxima registrada en este estudio $\left(17,5{ }^{\circ} \mathrm{C}\right)$, sugiere presencia de ASST o al menos una mezcla con esta masa de agua. El mes de febrero presenta características asociadas netamente a AESS en toda la columna de agua (Fig. 3). En superficie se registraron los valores de oxígeno más bajos de todo el período de estudio (Fig. 2). Finalmente el mes de abril registró características de ASSA en la capa superficial (Fig. 3), debido al mínimo de salinidad observado en los primeros metros. Más abajo la salinidad se incrementa a un valor máximo de 35,1 en la capa de 10 a $30 \mathrm{~m}$, lo que en conjunto con la elevada temperatura y alto contenido de oxígeno indican presencia de ASST. 


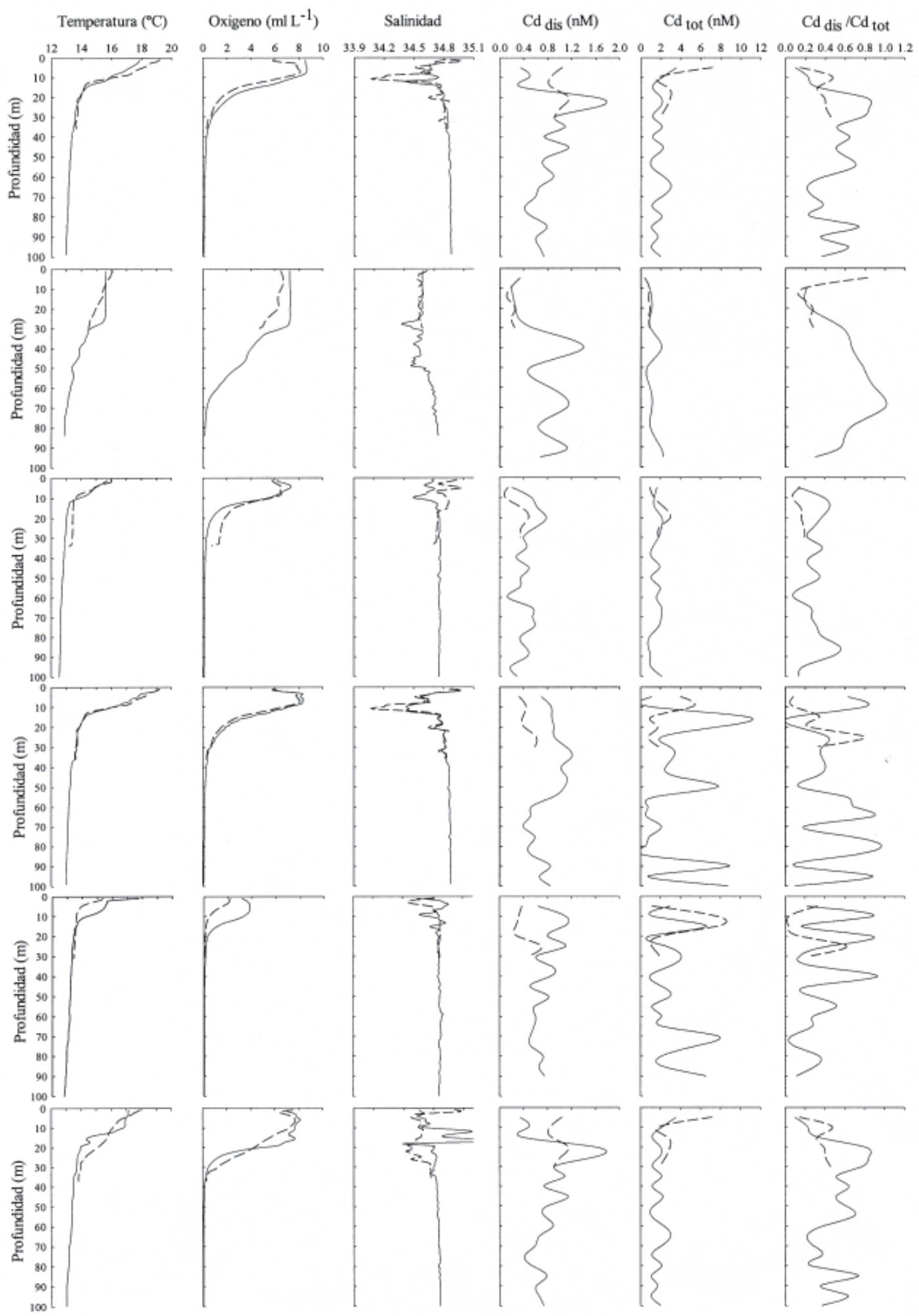

Fig. 2: Perfiles de temperatura, salinidad, oxígeno disuelto, $\mathrm{Cd}_{\mathrm{dis}}, \mathrm{Cd}_{\text {tot }}$ y razón $\mathrm{Cd}_{\mathrm{did}} / \mathrm{Cd}_{\text {tot }}$ en la bahía Mejillones, entre junio de 2002 y abril de 2003. La línea sólida corresponde a la Estación B y la línea punteada corresponde a la Estación A

Profiles of temperature, salinity, dissolved oxygen, $\mathrm{Cd}_{\mathrm{dis}}, \mathrm{Cd}_{\text {tot }}$ and $\mathrm{Cd}_{\mathrm{did}} / \mathrm{Cd}_{\text {tot }}$ ratio in Mejillones bay between June 2002 and April 2003. Solid line corresponds to the B station and dashed line to the A station. 

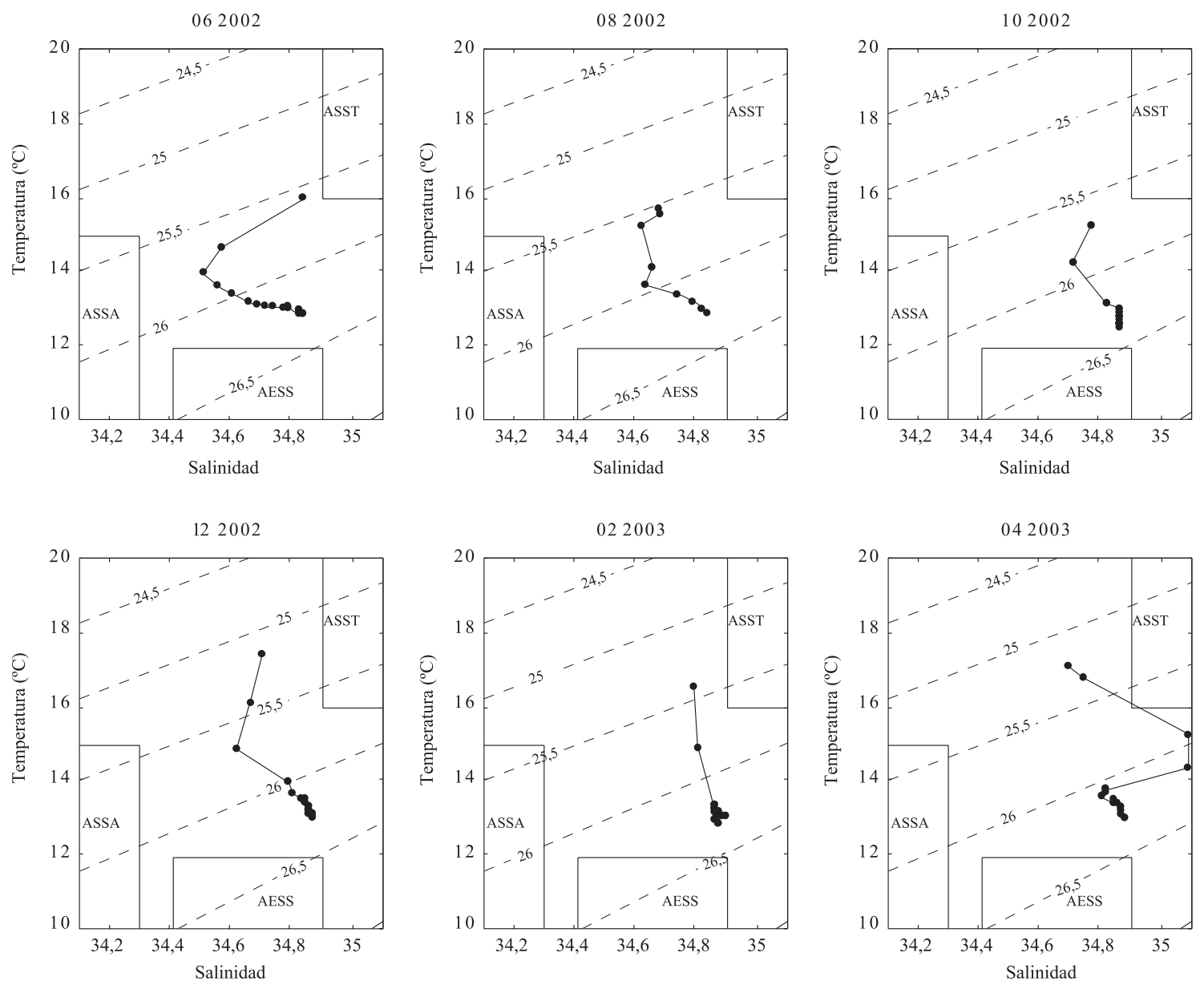

Fig. 3: Diagramas T-S para la serie de datos hidrográficos ubicados en la estación C de la Bahía de Mejillones del Sur. Período junio de 2002 a abril de 2003. Las áreas delimitadas incluyen en parte las características termosalinas de tres masas de agua: agua superficial subtropical (ASST), agua superficial subantártica (ASSA) y agua ecuatorial subsuperficial (AESS). Las características termosalinas son $16-24{ }^{\circ} \mathrm{C}$ y $34.9-35.3 ; 9-15{ }^{\circ} \mathrm{C}$ y $33-34.3 ; 8-12{ }^{\circ} \mathrm{C}$ y $34.4-34.9$, para el ASST, ASSA, y AESS, respectivamente.

Temperature-salinity diagrams for sampling months, performed with dates from pelagic station. Delimited areas consider thermo-haline characteristics of three water masses: subtropical superficial water (STSW), subantarctic superficial water (SASW) and Equatorial susuperficial water (ESSW). Thermo-haline characteristics are $16-24{ }^{\circ} \mathrm{C}$ and $34.9-35.3$; 9-15 ${ }^{\circ} \mathrm{C}$ and $33-34.3 ; 8-12{ }^{\circ} \mathrm{C}$ and 34.4-34.9, for STSW, SASW and ESSW, respectively.

La variabilidad de los parámetros físicoquímicos de la capa superficial medidas durante el período de estudio se muestra en la Fig. 4. La media anual de la temperatura, salinidad y oxígeno disuelto de las aguas superficiales son características de un año normal (no-El Niño), mientras que su rango de dispersión representa la influencia de la estacionalidad y la surgencia, sobre estos parámetros (Tabla 2). La temperatura superficial mínima registrada en Mejillones durante este período fue $14,5^{\circ} \mathrm{C}$, mientras que la máxima fue $19,1{ }^{\circ} \mathrm{C}$, ambas en la estación $\mathrm{A}$. El oxígeno disuelto varió entre $2,2 \mathrm{~mL} \mathrm{~L}^{-1}$ (Estación A) y $8,5 \mathrm{~mL} \mathrm{~L}^{-1}$ (Estación B).

Los valores medios de nitrato y fosfato fueron más altos en la Estación A que en la Estación B (Tabla 2). Estos valores fluctuaron entre 0,02 y $8,7 \mu \mathrm{M}$, para el caso del nitrato, y entre 1 y $2,7 \mu \mathrm{M}$, para el caso del fosfato. La fluctuación de los nutrientes durante el período de estudio mostró diferencias notorias. Mientras el nitrato presentó un patrón estacional bien definido (valores altos durante el período invernal y bajos durante el período 
TABLA 2

Estadística descriptiva de los parámetros medidos en este trabajo; $\mathrm{DE}=$ desviación estándar; nutrientes

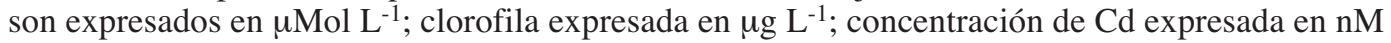

Descriptive statistics of all parameters measured in this study; DE = standard deviation; nutrients concentration indicated in $\mu \mathrm{Mol} \mathrm{L}{ }^{-1}$; chlorophyll-a concentration expressed in $\mu \mathrm{g} \mathrm{L}^{-1}$; Cd concentration expressed in $\mathrm{nM}$

\begin{tabular}{|c|c|c|c|c|c|c|}
\hline \multirow[t]{2}{*}{ Parámetro } & \multicolumn{2}{|c|}{ Media } & \multicolumn{2}{|c|}{$\mathrm{DE}$} & \multicolumn{2}{|c|}{ Rango } \\
\hline & Estacíón A & Estación B & Estación A & Estación B & Estación A & Estación B \\
\hline Temperatura $\left({ }^{\circ} \mathrm{C}\right)$ & 15,19 & 13,52 & 1,8 & 1,02 & $13,18-19,08$ & $12,55-17,55$ \\
\hline Oxígeno disuelto $\left(\mathrm{mL} \mathrm{L}^{-1}\right)$ & 4,06 & 1,45 & 2,17 & 2,43 & $0,08-8,36$ & $0,06-8,58$ \\
\hline Salinidad (psu) & 34,75 & 34,81 & 0,17 & 0,09 & $34,03-34,58$ & $34,51-35,08$ \\
\hline Clorofila $a\left(\mathrm{mg} \mathrm{L}^{-1}\right)^{*}$ & 3,31 & 2,82 & 2,16 & 1,86 & $0,79-6,36$ & $0,27-5,31$ \\
\hline Nitrato $(\mathrm{mM}) *$ & 2,38 & 0,96 & 3,53 & 1,41 & $0,06-8,7$ & $0,02-3,66$ \\
\hline Fosfato $(\mathrm{mM})^{*}$ & 1,68 & 1,25 & 0,62 & 0,27 & $1,00-2,70$ & $1,00-1,70$ \\
\hline Cadmio total & 2,17 & 2,21 & 1,9 & 1,9 & $0,41-7,78$ & $0,51-10,7$ \\
\hline disuelto & 0,42 & 0,59 & 0,3 & 0,35 & $0,09-1,15$ & $0,08-1,61$ \\
\hline
\end{tabular}

*Datos de superficie

estival), el fosfato registró sucesivas variaciones durante el año (Fig. 4).

La concentración de clorofila a registró un patrón de variación similar en ambas estaciones, con altos valores en primavera-verano, y bajos valores durante otoño-invierno (Fig. 4). La concentración media fue $3,31 \pm 2,16$ (Estación A) y $2,82 \pm 1,86$ (Estación C) (Tabla 2).

\section{Cadmio en la Bahía Mejillones}

Los valores de $\mathrm{Cd}_{\mathrm{dis}}$ y $\mathrm{Cd}_{\text {tot }}$ (integrando toda la columna de agua) durante el período de estudio presentaron una media de 0,42-0,59 nM (Estación A-Estación B) y 2,17-2,21 nM (Estación A-Estación B), respectivamente (Tabla 2). Los perfiles de $\mathrm{Cd}_{\mathrm{dis}}$ mostraron valores bajos en superficie, un máximo subsuperficial y una disminución hacia la zona más profunda. Con excepción de diciembre, estos niveles máximos coincidieron con la localización de la oxiclina y termoclina (Fig. 2). Los meses de diciembre y febrero presentaron las mayores concentraciones de $\mathrm{Cd}_{\mathrm{dis}}$ en toda la columna de agua. Los perfiles de $\mathrm{Cd}_{\text {tot }}$ fueron más irregulares, y aun cuando presentaron las mismas variaciones que la fracción disuelta, la disminución en la capa de agua de fondo no fue tan notoria (Fig. 2).

En la capa de agua superficial ambas estaciones presentaron un comportamiento similar en relación a la variabilidad de $\mathrm{Cd}_{\text {tot }} \mathrm{y}$ $\mathrm{Cd}_{\mathrm{dis}}$. Los valores se mantuvieron bajos entre junio y octubre, aumentando notoriamente a partir de este mes (Fig. 4). El rango de variación de $\mathrm{Cd}_{\text {tot }}$ y $\mathrm{Cd}_{\text {dis }}$ fue de $0,36-7,12 \mathrm{nM}$ y $0,09-0,98$ $\mathrm{nM}$, respectivamente. Los valores máximos se registraron en el mes de abril, en la Estación A.

\section{DISCUSIÓN}

Masas de agua durante el período 2002-2003

Los valores máximos y mínimos de temperatura observados a inicios del otoño y primavera respectivamente, se deberían al desfase temporal que generalmente tiene lugar entre la temperatura de la atmósfera y del océano (Gill 1982). A su vez, el valor mínimo de febrero se explicaría por el ingreso de aguas subsuperficiales, a la bahía, como consecuencia de procesos de surgencia en la zona de Punta Angamos.

Las masas de agua presentes al interior de la bahía Mejillones dependen del sistema de corrientes de Humboldt y sus masas de agua asociadas, de los procesos de surgencia de Punta Angamos en el extremo norte de la Península de Mejillones, de la variabilidad océano-climática ENSO, y de las características geográficas e hidrográficas de la bahía Mejillones (Srtub et al. 1998, Escribano et al. 2002). Esta interacción de factores hace que las propiedades conservativas y no conservativas de las masas de agua experimenten alteraciones $\mathrm{y} / \mathrm{o}$ modificaciones con respecto a las masas de agua originales ubicadas en la zona frente a la bahía. Entre estas modificaciones de las propiedades conservativas se destaca la tendencia a observar una mayor 
temperatura en la capa fótica al interior de la bahía, en relación a aquellas de la zona externa (Marín et al. 2001, Escribano et al. 2002, Marín et al. 2003). Esta situación fue observada durante el período de estudio especialmente en el caso del ASSA y ASST, ya que su
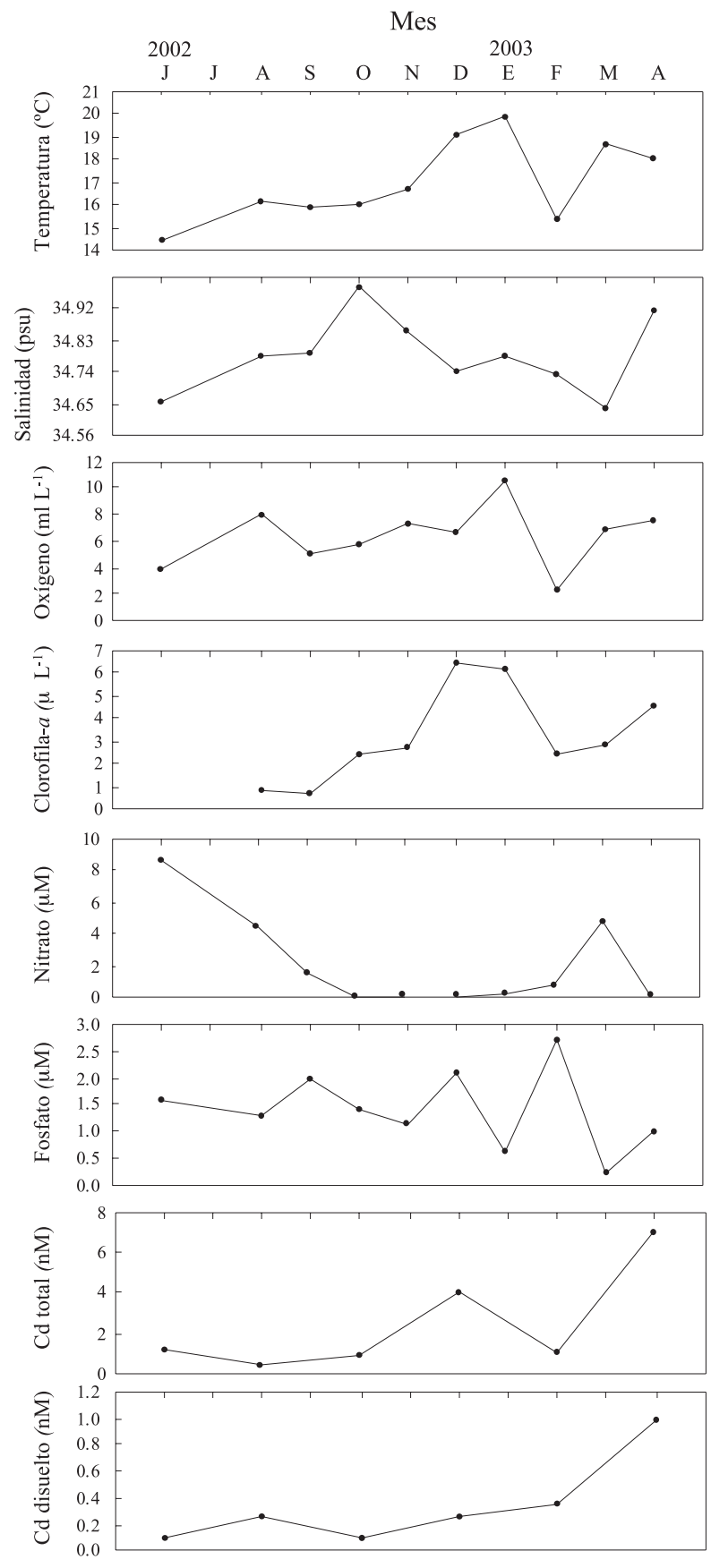

temperatura y salinidad se alteraron por los procesos de intercambio de calor con la atmósfera (en el primer caso) y como consecuencia de la mezcla con masas de agua subsuperficiales asociadas a la surgencia de Punta Angamos.
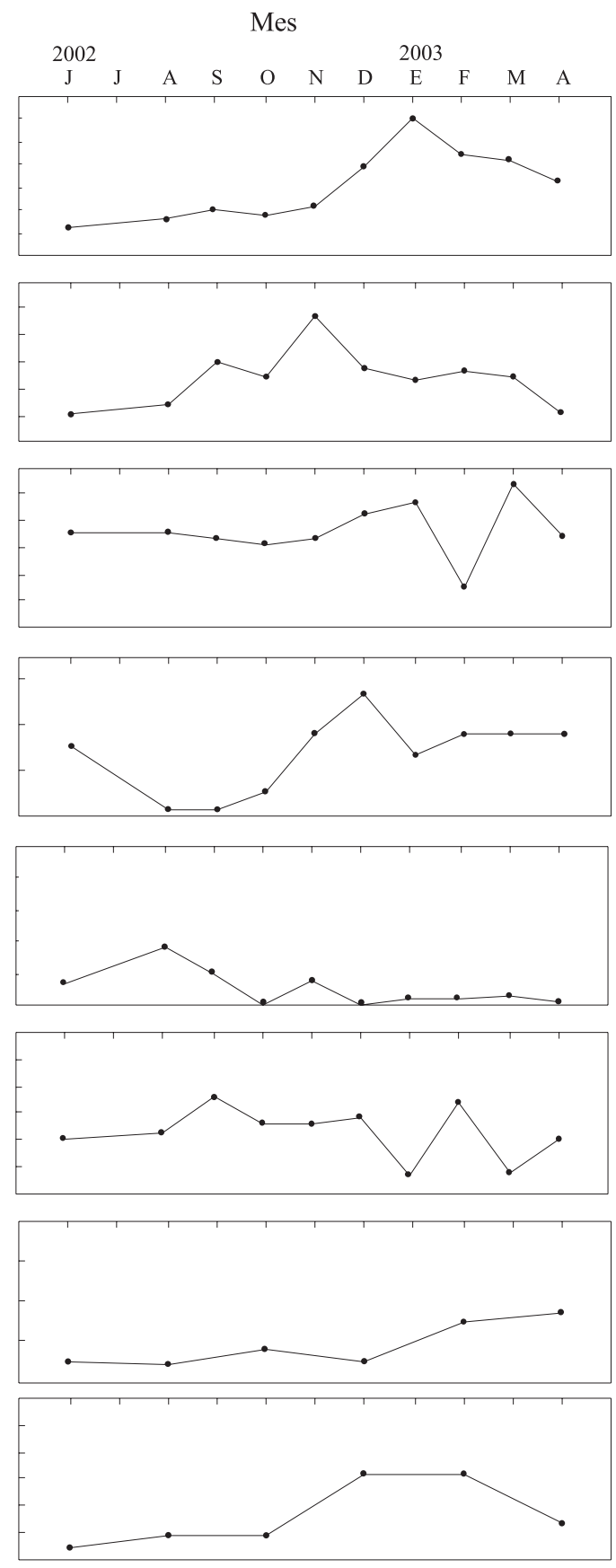

Fig. 4: Distribución temporal de temperatura, salinidad, oxígeno disuelto, nutrientes, clorofila y $\mathrm{Cd}$ en aguas superficiales de la bahía Mejillones.

Temporal distribution of temperature, salinity, dissolved oxygen, nutrients, chlorophill a and $\mathrm{Cd}$ in surface waters of Mejillones bay. 
Dentro del período de estudio, el mes de agosto presenta un comportamiento particular en cuanto a la distribución de propiedades físico-químicas de la columna de agua. Este mes registró un mayor contenido de oxígeno del orden de $7 \mathrm{~mL} \mathrm{~L}^{-1}$ hasta los $30 \mathrm{~m}$, el cual disminuye gradualmente con la profundidad hasta alcanzar condiciones micróxicas en los 70 m. El mayor contenido de oxígeno y la baja salinidad hacen suponer que se trata de una intrusión de agua con características de ASSA. Este período del año también coincide con una disminución en la intensidad de los vientos del suroeste (Escribano et al. 2004), lo cual podría ser responsable del ascenso de aguas de un nivel de menor profundidad con respecto al nivel donde se ubica el AESS, como se ha detectado en la zona frente a la Bahía de Mejillones (Sobarzo \& Figueroa 2001). Por el contrario, las características hidrográficas registradas en febrero hacen suponer la ocurrencia de un intenso evento de surgencia que produjo ascenso de aguas subsuperficiales en la zona externa a la Bahía. Esto estaría de acuerdo con lo observado en la literatura en cuanto a que en esta época del año se registran las mayores intensidades de viento del sur y suroeste (Escribano et al. 2004), lo cual favorece el ascenso de aguas correspondientes al nivel del AESS.

A pesar de que estos dos meses presentaron características bien definidas en cuanto a las masas de agua, los registros del período de estudio y datos anteriores no publicados indican que esta bahía se caracteriza por una permanente condición de mezcla de ASST. ASSA y AESS, además de una modificación de las propiedades que las caracterizan. Esta condición ejerce una importante influencia sobre el comportamiento de las sustancias químicas en los ambientes marinos, tal como ha sido sugerido, por ejemplo, para las aguas del Mar Mediterráneo (Yoon et al. 1999).

Variabilidad vertical de cadmio en Bahía Mejillones durante el período 2002-2003

El valor medio de $\mathrm{Cd}_{\text {dis }}$ en la capa de agua entre 0 y $100 \mathrm{~m}$ de profundidad en la bahía Mejillones fue de 0,59 $\pm 0,3 \mathrm{nM}$ (Tabla 2). Este valor es superior al reportado en otras zonas del Océano Pacífico. Por ejemplo, Abe (2001) reportó valores medios inferiores a 0,04 nM entre 0 y $150 \mathrm{~m}$ de profundidad en una zona de surgencia del Pacífico ecuatorial Este, mientras que Delgadillo-Hinojosa et al. (2001) encontraron concentraciones medias de 0,32 $\mathrm{nM}$ entre $\operatorname{los} 0$ y $100 \mathrm{~m}$ de profundidad en una zona de surgencia del Golfo de California.

El Cd es un metal no conservativo, y por lo tanto es afectado por procesos biológicos, ya sea vía "scavenging" y/o incorporación en el tejido biológico (Libes 1992, Brown et al. 1994, González-Dávila 1995). En general, el $\mathrm{Cd}$ es incorporado en el fitoplancton y reciclado más rápido que otros metales, lo que resulta en valores bajos en superficie y máximos muy próximos a ella (Noriki et al. 1985, Libes 1992, Calvert \& Pedersen 1993, Abe 2001, Delgadillo-Hinojosa et al. 2001). Esto genera perfiles muy bien definidos, con una marcada segregación vertical. En Mejillones, la distribución vertical de $\mathrm{Cd}_{\mathrm{dis}}$ corresponde a la descrita como tipo nutrientes, sin embargo es muy variable estacionalmente. Esta característica un tanto diferente de un perfil típico puede deberse a la condición de mezcla de masas de agua que caracteriza a la bahía Mejillones. Un argumento similar fue planteada por Delgadillo-Hinojosa et al. (2001) para explicar el comportamiento estacional del $\mathrm{Cd}$ en las aguas del Golfo de California. Por debajo de la capa superficial predominan los mecanismos de degradación de la materia orgánica, los cuales devuelven los nutrientes y micronutrientes (entre ellos el $\mathrm{Cd}$ ) al agua, hasta alcanzar una concentración máxima en la capa de agua subsuperficial. En la parte más profunda de la columna de agua, debido a la fuerte disminución del oxígeno disuelto (Fig. 2), la degradación no es tan eficiente de manera que los valores de $\mathrm{Cd}_{\mathrm{dis}}$ son generalmente bajos. Por otra parte, debido a que el $\mathrm{Cd}$ es considerado un metal redox-sensitivo (Calvert \& Pedersen 1993, Helz et al. 1996, Morford \& Emerson 1999, Bostick et al. 2000, Brown et al. 2000, Crusius \& Thomson 2000, Hammond et al. 2000, Zheng et al. 2000, Morford et al. 2001, Russell \& Morford 2001), los bajos valores de oxígeno presentes en la parte más profunda de la columna de agua de Mejillones ( $\left.<0,2 \mathrm{~mL} \mathrm{~L}^{-1}\right)$, favorecerían la formación $\mathrm{y}$ precipitación de complejos insolubles $\mathrm{CdS}$, tal como ha sido descrito para otras zonas (Kremling 1983, Pempkowiak et al. 2000). Sin embargo, este comportamiento ha sido 
establecido sobre la base de estudios de metales en sedimentos superficiales depositados bajo condiciones extremas (óxicas y anóxicas) (Veeh 1967, Bertine \& Turekian 1973, Jacobs \& Emerson 1982, Calvert \& Pedersen 1993, Colodner et al. 1993), más que en mediciones directas en la columna de agua (Nameroff et al. 2002). Mucho menos se conoce respecto de los efectos de condiciones intermedias (disóxicas/ micróxica) sobre el comportamiento de estos metales (Murray et al. 1995, Reu et al. 1997). Aún así, es un hecho que la modificación de las condiciones de oxigenación de la columna de agua es un proceso importante que influye en la solubilidad de los metales traza (entre ellos el
Cd) afectando su especiación y favoreciendo o limitando su exportación hacia los sedimentos de fondo (Pohl \& Hennings 2005). En el caso de Mejillones, el fuerte gradiente de oxígeno disuelto entre la superficie (óxica) y el fondo (micróxico) de la bahía (Fig. 2) ofrece condiciones ideales para evaluar la influencia de este parámetro sobre la distribución vertical de Cd. La Fig. 5A muestra la distribución de $\mathrm{Cd}_{\mathrm{dis}}$ de todo el período de estudio en la estación $\mathrm{B}$, en función del oxígeno disuelto de la columna de agua. Por su parte, la Fig. 5B muestra la distribución de $\mathrm{Cd}$ en sedimentos superficiales de la bahía Mejillones, en función del oxígeno disuelto del agua adyacente al piso
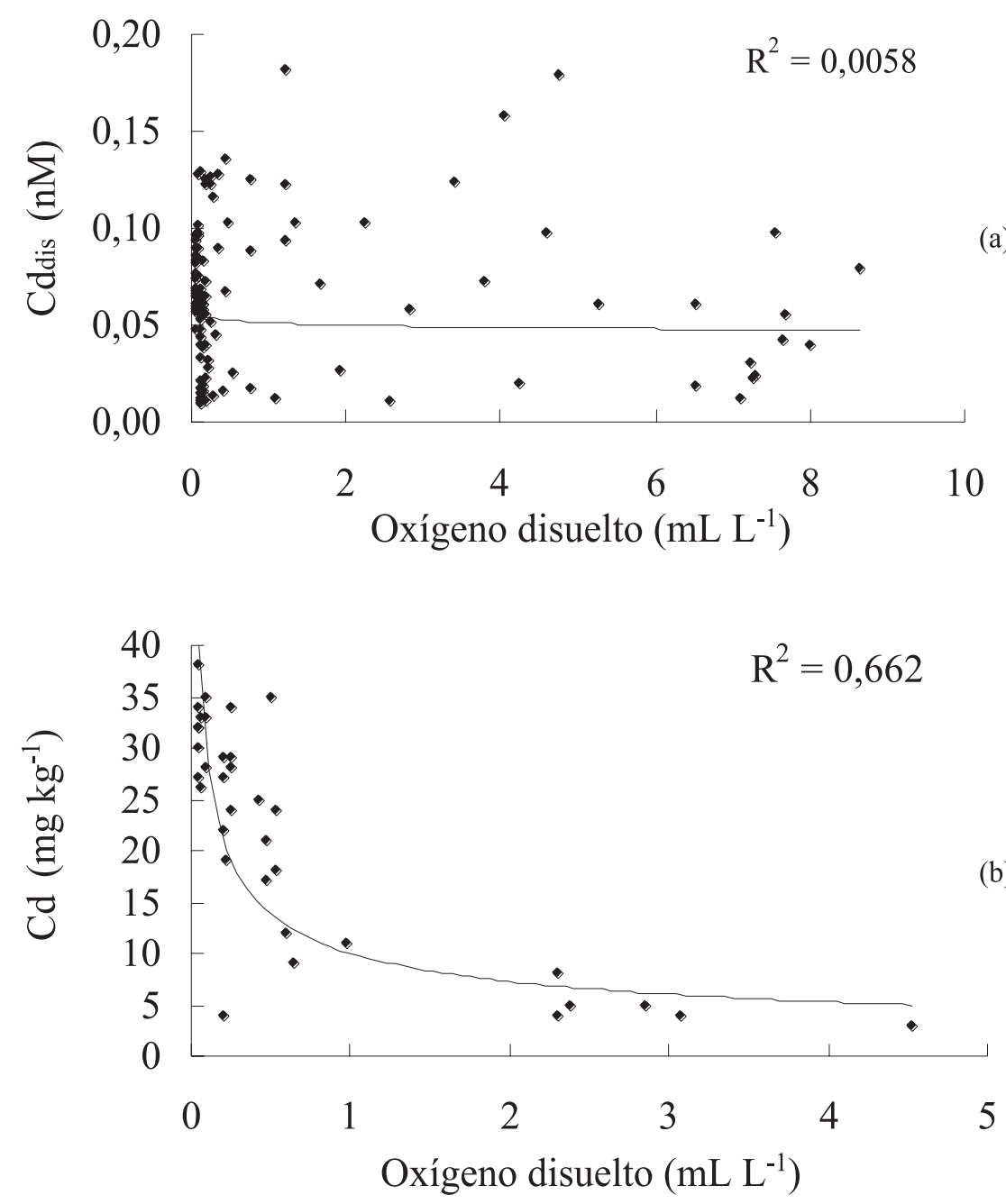

Fig. 5: (A) $\mathrm{Cd}_{\mathrm{ds}}$ versus oxígeno disuelto en la Estación B para todo el período de estudio. (B) Cd versus oxígeno disuelto en sedimentos superficiales de la bahía Mejillones (ver Valdés et al. 2004).

(A) $\mathrm{Cd}_{\mathrm{dis}}$ versus disolved oxygen in B station for all sampling period. (B) Cd versus disolved oxygenin surface sediment of Mejillones bay (see Valdés et al. 2004). 
marino, en cada punto de muestreo (ver Valdés 2004). El primero de estos gráficos muestra una distribución totalmente irregular de la concentración de $\mathrm{Cd}_{\mathrm{dis}} \mathrm{y}$ ninguna correlación con el oxígeno disuelto. Por el contrario, la distribución de $\mathrm{Cd}$ en los sedimentos superficiales muestra una correlación significativa $(\mathrm{P}<0,001)$ con el oxígeno disuelto. Estos datos indican que en Mejillones la condición micróxica de las aguas subsuperficales no es suficiente para remover Cd desde la columna de agua hacia los sedimentos de fondo, ya que no se observó una disminución significativa de $\mathrm{Cd}_{\mathrm{dis}}$ asociada a la disminución de oxígeno. Estos mismos antecedentes $\left(\mathrm{Cd}_{\mathrm{dis}}\right.$ versus oxígeno disuelto) fueron utilizados para concluir un comportamiento similar de este metal en las aguas del Pacífico tropical, frente a México (Nameroff et al. 2002). Por el contrario, en la cuenca de Gotland (Mar Báltico) se pudo establecer que las aguas subsuperficiales anóxicas favorecían la precipitación y remoción de $\mathrm{Cd}_{\text {dis }}$ desde la columna de agua hacia los sedimentos de fondo (Pohl \& Hennings 2005). Por su parte, los resultados de $\mathrm{Cd}$ en los sedimentos superficiales (Fig. 5B) indican que el oxígeno ejerce una fuerte influencia sobre la mantención de este metal inmovilizado en los sedimentos, bajo condiciones mínimas de oxígeno. Se concluye que el mecanismo preponderante que transfiere $\mathrm{Cd}$ desde la columna de agua hacia los sedimentos sería el flujo biogénico más que el oxígeno disuelto, mientras que este último factor sería el responsable de la preservación de este metal en los sedimentos de fondo. De esta manera, la concentración de $\mathrm{Cd}$ no puede ser utilizada para distinguir entre condiciones óxicas y micróxicas en la columna de agua de Mejillones.

La mezcla de aguas al interior de la bahía Mejillones dificulta estimar un contenido de $\mathrm{Cd}$ característico para cada masa de agua identificada. Sin embargo, el mes de febrero, caracterizado por el predominio de AESS a lo largo de toda la columna de agua (Fig. 3), permiten a lo menos sugerir que esta masa de agua presenta una concentración de $\mathrm{Cd}_{\mathrm{tot}} \mathrm{y} \mathrm{Cd}_{\mathrm{dis}}$ en torno de los valores medios registrados en este mes; 3,29 y $0,73 \mathrm{nM}$, respectivamente. Particularmente, la concentración media de $\mathrm{Cd}_{\text {tot }}$ es la más alta de todo el período de estudio
Debido a que el $\mathrm{Cd}_{\mathrm{dis}}$ es solo una fracción de todo el Cd presente en el agua de mar se utilizó la razón $\mathrm{Cd}_{\text {dis }} / \mathrm{Cd}_{\text {tot }}$ para comparar la forma de Cd predominante a lo largo de la columna de agua. Así, valores superiores a 0,5 indican un predominio de la fracción disuelta, y valores inferiores a este indican un predominio de la fracción particulada. Los resultados de Mejillones muestran valores medios de $0,25 \mathrm{y}$ 0,36 para las estaciones A y B, respectivamente, lo que evidencia que la forma predominante durante el período de estudio fue $\mathrm{Cd}$ particulado. Esta situación ha sido descrita como característica para el océano Pacífico (Saager et al. 1992). Sin embargo, todos los perfiles muestran niveles de profundidad en los que la fracción disuelta fue mayoritaria, representando en algunos casos a más del $90 \%$ de todo el $\mathrm{Cd}$ presente en el agua (Fig. 2). Estos resultados permiten identificar dos períodos durante la época de estudio (Fig. 2). Un primer período comprendido por junio de 2002 y febrero-abril de 2003 durante el cual se evidenció un mayor predominio de la fracción disuelta en la capa de agua superficial que en la capa de fondo, y una zona de transición entre estas dos condiciones ubicada a nivel de termo-oxiclina. El segundo período corresponde a los meses de agosto, octubre y diciembre, con una situación contraria. Aquí el predominio de la fracción de $\mathrm{Cd}_{\text {dis }}$ fue mayor en la capa de agua de fondo. El predominio de $\mathrm{Cd}_{\text {dis }}$ registrado durante este segundo período en las aguas de fondo de la bahía en comparación con las aguas superficiales, puede ser el resultado de los procesos de circulación de meso-escala (i.e., surgencia) que vinculan a la bahía con el centro de surgencia de Punta Angamos. Esta condición hidrodinámica es característica de la mayoría de las bahías con alineación norte-sur ubicada sobre la costa del Pacífico sureste (Ahumada 2002). En esta zona, los eventos de surgencia transportan AESS hacia la superficie, las cuales presentan un leve enriquecimiento en $\mathrm{Cd}_{\text {dis }}$ (Ahumada \& Contreras 1997). De esta manera, la advección subsuperficial de AESS reforzada durante este período puede significar un ingreso adicional de $\mathrm{Cd}_{\mathrm{dis}}$ al interior de la bahía, generando un predominio de la fracción disuelta en las aguas de fondo. En este patrón de variación, el mes de febrero corresponde a una transición hacia una condición invernal mientras que el mes de agosto lo es hacia una condición estival. 
Dinámica superficial del cadmio en Bahía Mejillones

Los resultados de las variables físico-químicas de superficie (temperatura, salinidad, oxígeno disuelto, nutriente, clorofila $a$ ) son similares a las reportadas por otros autores, para diferentes períodos considerados como no-El Niño (Navea \& Miranda 1980, Rodríguez et al. 1991, Marín et al. 1993, Rodríguez \& Escribano 1996, Marín \& Olivares 1999, Marín et al. 2003).

En las aguas superficiales, la concentración de $\mathrm{Cd}_{\text {tot }}$ medida en Mejillones es superior a la reportada en otras regiones oceánicas caracterizadas por el desarrollo de eventos de surgencia (Tabla 3 ). La concentración de $\mathrm{Cd}_{\mathrm{dis}}$ registrada en superficie en Mejillones varió entre 0,09 y $0,98 \mathrm{nM}$, valores mayores a los registrados en el Océano Atlántico, Mar Báltico y el Pacífico tropical este (ver referencias en Tabla 3). Particularmente, Takesue et al (2004) reportaron valores de $\mathrm{Cd}_{\mathrm{dis}}$ de entre 0,1 y 1,0 nM, durante el período 1997-2000, en la localidad de Isla Santa María, Península de Mejillones (Fig. 1), muy similar a lo reportado en este trabajo para la bahía Mejillones. Si bien el período el trabajo de Takesue et al. (2004) incluye al evento El Niño 1997-98, los autores indican que, al menos para esta localidad, la concentración de $\mathrm{Cd}_{\mathrm{dis}}$ no responde significativamente al desarrollo de este evento.
La presencia de $\mathrm{Cd}$ en las aguas costeras superficiales es comúnmente explicada por dos mecanismos: aguas de fondo transportadas hacia la superficie por eventos de surgencia, y/o material litogénico transportado desde el continente a través de los ríos y la atmósfera. Al respecto, Bruland (1980) calculó que el flujo atmosférico de Cd hacia el Pacífico es cerca de un orden de magnitud menor que el flujo por mezcla vertical de la columna de agua. En el caso de Mejillones, la condición desértica de esta región descarta el transporte de $\mathrm{Cd}$ litogénico vía cursos de ríos. Además, Valdés \& Ortlieb (2001) y Valdés (2004) correlacionaron la concentración de $\mathrm{Cd}$ con la concentración de aluminio (un marcador de fuente litogénica, según Schropp et al. 1990, Wehausen \& Brumsack 1998, entre otros) en sedimentos superficiales y testigos de Mejillones, y concluyeron que la presencia de $\mathrm{Cd}$ en los sedimentos de fondo no se explica por el aporte continental. Las fuentes contaminantes también pueden aportar cantidades significativas de metales a las aguas costeras (Pempkowiak et al. 2000). Sin embargo, los bajos niveles de $\mathrm{Cd}_{\text {tot }}$ encontrados en las aguas superficiales (los cuales son típicos del Océano Pacífico, Tabla 2), y los valores de $\mathrm{Cd}_{\mathrm{dis}}$, idénticos a los registrados por Takesue et al. (2004) en Isla Santa María, un ambiente libre de contaminación industrial,

TABLA 3

Concentración media o rango de Cd total y disuelto en aguas superficiales de diferentes zonas oceánicas. Los valores reportados por Takesue et al. (2004) corresponden a la costa chilena

Mean or rank, of total and disolved Cd concentration in surface waters of different oceanic areas. Values reported by Takesue et al. (2004) correspond to the Chilean coast

\begin{tabular}{|c|c|c|c|}
\hline Zona & $\mathrm{Cd}_{\text {tot }}(\mathrm{nM})$ & $\mathrm{Cd}_{\mathrm{dis}}(\mathrm{nM})$ & Fuente \\
\hline Atlántico noreste & 0,23 & - & Danielson et al. (1985) \\
\hline Aguas costeras portuguesas & $0,04-0,25$ & - & Cotté-Krief et al. (2000) \\
\hline Margen de California-México & $0,03-0,166$ & - & Segovia-Zavala et al. (1998) \\
\hline Atlántico (transecta a los $\left.23^{\circ} \mathrm{S}\right)$ & $0,002-0,1$ & - & Kremling (1985) \\
\hline Mar Báltico, Polonia & - & $0,009-0,12$ & Pempkoviak et al. (2000) \\
\hline Pacífico tropical noreste & - & 0,26 & Nameroff et al. (2002) \\
\hline Océano Índico, Omán & - & 0,08 & Saager et al. (1992) \\
\hline El Quisco $\left(33,4^{\circ} \mathrm{S}\right)$ & - & $0,2-0,6$ & Takesue et al. (2004) \\
\hline Coliumo $\left(36,5^{\circ} \mathrm{S}\right)$ & - & $0,1-0,8$ & Takesue et al. (2004) \\
\hline Isla Santa María $\left(23,4^{\circ} \mathrm{S}\right)$ & - & $0,1-1,0$ & Takesue et al. (2004) \\
\hline Bahía Mejillones $\left(23^{\circ} \mathrm{S}\right)$ & $0,36-7,12$ & $0,09-0,98$ & Este estudio \\
\hline
\end{tabular}


descartan este tipo de aportes. Por otra parte, Valdés et al. (2005) estudiaron la distribución superficial de diferentes metales en los sedimentos de la bahía Mejillones, incluido el $\mathrm{Cd}$, concluyendo que no hay evidencias de contaminación en este ambiente costero. De esta forma, se confirmaría que el Cd presente en las aguas y sedimentos de la bahía Mejillones tiene un origen más bien marino, al menos hasta el momento de realizarse este estudio.

La distribución de metales en las aguas superficiales se ve altamente influenciada por los procesos oceanográficos que en ella ocurren (Yoon et al. 1999). Como una forma de identificar los factores que determinan las características hidrográficas superficiales de Mejillones, y siguiendo el procedimiento utilizado por Segovia-Zavala et al. (1998), se aplicó un análisis de Componente Principales a todas las variables medidas en superficie. Este análisis evidenció que dos componentes explican el $66 \%$ de la varianza total de los datos, durante el período de estudio (Tabla 4). El primer componente indica una relación directa entre la temperatura, la concentración de clorofila $a$ y el contenido de $\mathrm{Cd}$, con un $43,2 \%$ de la varianza total. El segundo componente destaca la relación inversa entre el oxígeno disuelto y el contenido de fosfato, con un $22,8 \%$ de la varianza total. Esta correlación negativa entre oxígeno y fosfato es característica de eventos de surgencia costera en esta bahía (Rodríguez et al. 1991), mientras que la clorofila $a$ es usada comúnmente como un marcador de biomasa fitoplanctónica (Stephens et al. 1997, Sun \& Dai 2005). De esta manera los datos de este estudio indican que la distribución de $\mathrm{Cd}$ en las aguas superficiales de Mejillones es gobernada, principalmente, por factores biológicos (producción de biomasa fitoplanctónica), más que por los factores físicos (surgencia). Esta interpretación concuerda con la reportada por, Takesue et al. (2004) quienes realizaron un muestreo quincenal de $\mathrm{Cd}_{\text {dis }}$ en agua superficiales en la isla Santa María (al oeste de Península Mejillones, Fig. 1), concluyendo que no hay argumentos suficientes para indicar que, en esta localidad, la variabilidad temporal de la concentración de este metal sea gobernada por los eventos de surgencia costera.
TABLA 4

Análisis de componentes principales para las variables medidas en superficie en Mejillones entre junio del 2002 y abril del 2003

Principal component analysis of parameters measured in Mejillones bay, between June 2002 and April 2003

\begin{tabular}{lcc}
\hline Variable & $\begin{array}{c}\text { Componente } \\
\text { principal } 1\end{array}$ & $\begin{array}{c}\text { Componente } \\
\text { principal 2 }\end{array}$ \\
\hline Temperatura & $-0,469$ & 0,056 \\
Oxígeno & 0,013 & 0,676 \\
Salinidad & $-0,229$ & 0,040 \\
Clorofila $a$ & $-0,459$ & $-0,038$ \\
Nitrato & 0,378 & 0,219 \\
Fosfato & $-0,069$ & $-0,670$ \\
Cadmio total & $-0,443$ & 0,164 \\
Cadmio disuelto & $-0,416$ & 0,115 \\
Varianza & 0,432 & 0,228 \\
Varianza acumulada & 0,432 & 0,660 \\
\hline
\end{tabular}

Sin embargo, se debe considerar que la frecuencia de muestreo utilizada en este estudio dificulta la identificación de eventos de surgencia que son de corta duración, y por lo tanto, esta conclusión es válida para una escala temporal estacional. En una escala de tiempo menor, Marín et al. (1993), compararon el desarrollo de eventos de surgencia en Punta Angamos con las condiciones internas de la bahía, durante el período primaveral, utilizando un intervalo de muestreo de 10 días. Los autores concluyeron que la dinámica biológica primaveral diaria de Mejillones es controlada por la surgencia de Punta Angamos. En un trabajo posterior, Marín \& Olivares (1999) confirmaron que los procesos más importantes en el control de la productividad primaria de Mejillones operan en escalas inferiores a la estacional, pero que cuando se elimina esta variabilidad de mayor frecuencia emergen patrones más bien biológicos (i.e., clorofila) como representativos del régimen oceanográfico de esta bahía.

Relación entre $\mathrm{Cd}_{\text {dis }}$ y $\mathrm{PO}_{4}$ en las aguas superficiales de Mejillones

En el océano, el $\mathrm{Cd}$ y el $\mathrm{PO}_{4}$ generalmente exhiben una relación lineal, lo cual es importante para entender los procesos biogeoquímicos que ocurren en el ecosistema 
marino (Sañudo-Wilhelmy et al. 2002). Autores como Abe \& Matsunaga (1988), Boyle et al. (1985), Van Geen et al. (1992), Van Geen \& Husby (1996) y Takesue \& Van Geen (2002) indican que tanto la distribución vertical como la distribución en las aguas superficiales de $\mathrm{Cd}$ se correlaciona significativamente con la de fosfato. Los resultados obtenidos en Península Mejillones (Fig. 1) no son equivalentes. Mientras que en Isla Santa María (zona externa de la península) ambos parámetros mostraron una correlación significativa (Takesue et al. 2004) en Bahía Mejillones estos parámetros no se correlacionaron. Esta situación muestra claras diferencias entre la zona externa de la península (lugar en donde se ubica el foco de surgencia) y la bahía propiamente tal, lo cual puede ser un reflejo de la condición de "sombra de surgencia" que Marín et al. (2003) le atribuyen a esta bahía. Cabe destacar que esta falta de correlación entre el $\mathrm{Cd}$ y el $\mathrm{PO}_{4}$ también ha sido reportada en otras zonas costeras como el margen noroeste de Baja California (Segovia-Zavala et al. 1998) y las aguas superficiales del Atlántico (Kremling 1985).

Se ha establecido que la razón $\mathrm{Cd}_{\mathrm{dis}} / \mathrm{PO}_{4}$ no difiere mucho en diferentes zonas oceánicas y, en el caso del Océano Pacífico, fluctúa entre 0,1 y 0,3 (Tabla 5). Los valores de Mejillones están dentro del rango típico del Océano Pacífico, y son el resultado de condiciones hidrográficas regionales y estacionales específicas que afectan los procesos biológicos asociados al ciclo de estos elementos, tal como ha sido reportado para otras zonas (Pohl et al. 1993, Nolting \& de Baar 1994, Loscher et al. 1998). Takesue et al. (2004) compararon los valores de $\mathrm{Cd}_{\mathrm{dis}} / \mathrm{PO}_{4}$ de la costa este del Pacífico sur con la del Pacífico norte, y encontraron una razón menor en el primer caso. Los autores concluyen que esta situación puede ser la resultante de diferencias en la composición de las masas de agua de fondo que originan la surgencia, ya sea debido a la condición de metal redox del $\mathrm{Cd}$ y/o al comportamiento del fósforo en la interfase aguasedimento.

La Fig. 6 muestra la variación de la razón $\mathrm{Cd}_{\mathrm{dis}} / \mathrm{PO}_{4}$ en las aguas superficiales de Mejillones durante el período de estudio. Se observa que esta razón mantiene valores bajos durante el invierno y aumenta hacia el verano. Este patrón de comportamiento puede ser explicado por la variación de la productividad biológica de la bahía. Durante el invierno, la baja productividad (ver gráfico de clorofila en Fig. 4) mantiene valores altos de $\mathrm{PO}_{4}$ sin utilizar, en la capa de agua superficial (Fig. 4). Durante el verano, la productividad aumenta y se genera una mayor eficiencia en la utilización de $\mathrm{PO}_{4}$ por parte del fitoplancton. Esto puede provocar una remoción más rápida de este nutriente que de $\mathrm{Cd}$, en el agua superficial, generando un aumento de la razón $\mathrm{Cd}_{\mathrm{dis}} / \mathrm{PO}_{4}$ (Fig. 6). Una explicación alternativa (o complementaria) es que el proceso de remineralización de $\mathrm{Cd}$ debido a la degradación de materia orgánica, ocurre más rápido que el de $\mathrm{PO}_{4}$, lo que genera un aumento del $\mathrm{Cd}$ disponible en aguas superficiales (Fig. 4). La combinación de estos mecanismos puede explicar el patrón de variación de la razón $\mathrm{Cd}_{\mathrm{dis}} / \mathrm{PO}_{4}$ en la bahía Mejillones, tal como ha sido sugerido para el Golfo de California por Delgadillo-Hinojosa (2001).

TABLA 5

Razón $\mathrm{Cd}_{\mathrm{dis}} / \mathrm{PO}_{4}$ en diferentes ambientes oceánicos

$\mathrm{Cd}_{\text {dis }} / \mathrm{PO}_{4}$ ratio in different oceanic environments

\begin{tabular}{lcc}
\hline Zona & $\mathrm{Cd}_{\mathrm{dis}} / \mathrm{PO}_{4}(\mathrm{nM} / \mu \mathrm{M})$ & Fuente \\
\hline Océano Índico & 0,16 & Saager et al. (1992) \\
Pacífico tropical este & 0,31 & Nameroff et al. (2002) \\
Pacífico ecuatorial oeste & 0,109 & Abe (2001) \\
Pacífico noreste & 0,31 & Bruland et al. (1978) \\
Mar de Weddell & 0,53 & Sañudo-Wilhelmy et al. (2002) \\
Isla Santa María $\left(23,4^{\circ} \mathrm{S}\right)$, Península de Mejillones & 0,27 & Takesue et al. (2004) \\
Bahía de Mejillones & 0,25 & Este estudio \\
\hline
\end{tabular}




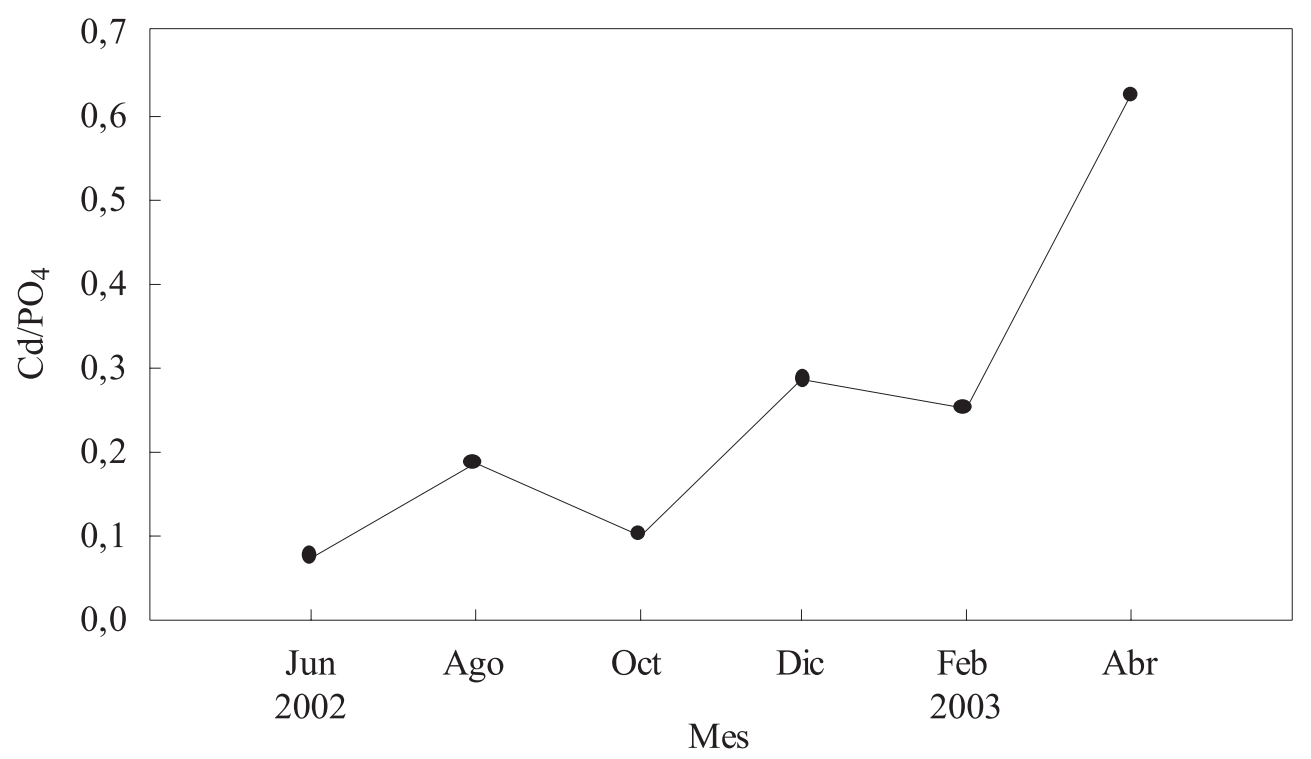

Fig. 6: Variabilidad estacional de la razón $\mathrm{Cd}_{\mathrm{dis}} / \mathrm{PO}_{4}(\mathrm{nM} / \mu \mathrm{M})$ en aguas superficiales de la bahía Mejillones durante el período 2002-2003.

Seasonal variability of $\mathrm{Cd} / \mathrm{PO}_{4}(\mathrm{nM} / \mu \mathrm{M})$ ratio in surface waters of Mejillones bay during 2002-2003.

\section{CONCLUSIONES}

El rango de cadmio total y cadmio disuelto medido durante el período 2002-2003 en las aguas de la bahía Mejillones fue 0,41-10,7 y 0,08-1,61 nM, respectivamente. Las variables oceanográficas evidenciaron un ciclo anual noEl Niño, caracterizado por altos valores de nutrientes durante el periodo invernal y bajos valores durante el período estival. La variabilidad estacional de las masas de agua en la capa superficial permite establecer predominio de ASSA en la capa superficial durante los meses de invierno junio-agosto, AESS en el mes de febrero y ASST en el mes de abril en la capa comprendida en entre 10-30 $\mathrm{m}$. En general estas masas de agua presentaron diferentes grados de mezcla durante los meses muestreados, lo cual dificulta asignar un valor de contenido de $\mathrm{Cd}$ característico para cada masa de agua identificada. Aún así, y basado en los resultados del mes de febrero se sugiere que las AESS presentan una concentración de $\mathrm{Cd}_{\text {tot }}$ y $\mathrm{Cd}_{\mathrm{dis}}$ en torno de 3,29 y $0,73 \mathrm{nM}$, respectivamente.

No se encontró una influencia significativa del oxígeno disuelto sobre la disponibilidad de $\mathrm{Cd}$, lo que sugiere que este metal no sería un buen marcador de condiciones micróxicas de la columna de agua, al menos en esta bahía. Los perfiles de $\mathrm{Cd}$ presentaron una distribución vertical clásica de un micronutriente. Sin embargo estos perfiles presentaron leves diferencias que pueden ser atribuidas a la mezcla de masas de agua al interior de la bahía. La variabilidad estacional de la concentración de $\mathrm{Cd}$ en las aguas superficiales de Mejillones sería regulada, al menos estacionalmente, por la producción de biomasa fitoplanctónica.

La razón $\mathrm{Cd}_{\mathrm{dis}} / \mathrm{PO}_{4}$ de las aguas de Mejillones son similares a las reportadas por otros estudios para la costa de Chile, mientras que su variabilidad estacional estaría relacionada con el balance entre la utilización de $\mathrm{Cd}$ y fosfato en las aguas superficiales y su remineralización en las aguas subsuperficales de la bahía.

\section{AGRADECIMIENTOS}

Este estudio fue financiado con el proyecto PEI 1340-02, de la Universidad de Antofagasta, y la UR 055 Paleotropique, del Institut de Recherche pour le Developpement (Francia). Los autores agradecen el apoyo de Freddy Rabasté y Keyla Majluf en la recolección de las muestras, y análisis de nutrientes y clorofila. 


\section{LITERATURA CITADA}

ABE K (2001) Cd in the western equatorial Pacific. Marine Chemistry 74: 197-211.

ABE K \& K MATSUMAGA (1988) Mechanism controlling $\mathrm{Cd}$ and $\mathrm{PO}_{4}$ concentration in Funka Bay, Japan. Marine Chemistry 23: 145-152.

AHUMADA R \& S CONTRERAS (1997) Hipótesis sobre el contenido de cadmio en dos bahías de Chile Central. Resúmenes XVII Congreso de Ciencias del Mar. Universidad de Chile, Santiago, Chile. p 76

AHUMADA R (2002) Concepción bay - A tectonic embayment on the south eastern Pacific coastline: a case study. En: Castilla JC \& JL Langlier (eds) The oceanography and ecology of the nearshores and bays in Chile: 67-93. Ediciones Universidad Católica de Chile, Santiago, Chile.

BERTINE K \& K TUREKIAN (1973) Molybdenum in marine deposits. Geochimica et Cosmochimica Acta 37: 1415-1434

BOSTICK B, S FENDORF \& M FENDORF (2000) Disulfide disproportionation and CdS formation upon cadmium sorption on $\mathrm{FeS}_{2}$. Geochimica et Cosmochimica Acta 64: 247-255.

BOYLE E (1988) Cadmium: chemical tracer of deepwater paleoceanography. Paleoceanography 3: 471-489.

BOYLE E, S HAPNICK, X BAI \& A SPIVACK (1985) Trace metals enrichment in the Mediterranean Sea. Earth and Planetary Sciences Letter 74: 405-419.

BROWN J, A COLLING, D PARK, J PHILLIPS, D ROTHERY \& J WRIGHT (1994) Ocean Chemistry and Deep-Sea sediments. The Open University, Pergamon Press. Oxford, United Kingdom. 133 pp.

BROWN E, L CALLONEC \& CH GERMAN (2000) Geochemical cycling of redox-sensitive metals in sediments from lake Malawi: a diagnostic paleotracer for episodic changes in mixing depth. Geochimica et Cosmochimica Acta 64: 3515-3523.

BRULAND K, G KNAUER \& J MARTIN (1978) cadmium in the northeast Pacific waters. Limnology and Oceanography 23: 618-625.

BRULAND K (1980) Oceanographic distribution of cadmium, zinc, nickel and copper in the north Pacific. Earth and Planetary Sciences Letter 47: 176-198.

BRULAND K, K ORINAS \& J COWEN (1994) Reactive trace metals in the stratified central north Pacific. Geochimica et Cosmochimica Acta 58: 3171-3182.

CALVERT S \& T PEDERSEN (1993) Geochemistry of recent oxic and anoxic marine sediments: Implications for the geological records. Marine Geology 11: 67-88.

COLODENER D, J SACH, G RAVIZZA, K TUREKIAN, J EDMOND \& E BOYLE (1993) The geochemical cycle of rhenium: a reconnaissance. Earth and Planetary Science Letter 117: 205-221.

COTTE-KRIEF M, C GUIEU, A THOMAS \& J MARTIN (2000) Sources of $\mathrm{Cd}, \mathrm{Cu}, \mathrm{Ni}$ and $\mathrm{Zn}$ in the Portuguese coastal waters. Marine Chemistry 71: 199-214.

CRUSIUS J \& J THOMSON (2000) Comparative behavior of authigenic $\mathrm{Re}, \mathrm{U}$, and Mo during reoxidation and subsequent long-term burial in marine sediments. Geochimica et Cosmochimica Acta 64: 2233-2242.

DANIELSON L, B MAGNUSON \& S WEATERLUND (1985) Cadmium, copper, iron nickel and zinc in the northeast Atlantic Ocean. Marine Chemistry 17: 23-41.

DE BAAR H P SAAGER R NOLTING \& J VANDERMEER (1994) Cadmium versus phosphate in the world ocean. Marine Chemistry 46: 261-281.

DELGADILLO-HINOJOSA F, J MACÍAS-ZAMORANO, J SEGOVIA-ZAVALA \& S TORRES-VALDÉS (2001) Cadmium enrichment in the Gulf of California. Marine Chemistry 75: 109-122.

ELBAZ-POULICHET F \& J MARTIN (1987) Dissolved $\mathrm{Cd}$ behavior in some selected French and Chinese estuaries. Consequences on Cd supply to the ocean. Marine Chemistry 22: 125-136.

ESCRIBANO R (1998) Population dynamics of Calanus chilensis in the Chilean eastern boundary Humboldt Current. Fisheries Oceanography 7: 245-251

ESCRIBANO R \& HIDALGO P (2000) Spatial distribution of copepods in the north of the Humboldt current region off Chile during coastal upwelling. Journal of the Marine Biology Association UK 80: 283-290.

ESCRIBANO R, V MARÍN P HIDALGO \& G OLIVARES (2002) Physical-biological interactions. In the pelagic ecosystem of the nearshore zone of the northern Humboldt Current System. In: J. C: Castilla \& J. Largier (eds) The Ocanography and Ecology of the nearshore and bays in Chile: 145-175. Proceedings of the international symposium on Linkages and Dynamics of Coastal System: Open coast and Embayment. Ediciones Universidad Católica de Chile, Santiago, Chile.

ESCRIBANO R, S ROSALES \& JL BLANCO (2004) Understanding upwelling circulation off Antofagasta (northern Chile): A three - dimensional numerical-modelling approach. Continental Shelf Research 24: 37-53.

ESTELA J, C TOMÁS, A CLADERA \& V CERDÀ (1995) Potentiometric stripping analysis: a review. Critical Reviews in Analytical Chemistry 25: 91-141.

GILL AE (1982) Atmosphere-ocean dynamics. International Geophysics Series 30. Academic Press, London, United Kingdom. 662 pp.

GONZÁLEZ HE, G DANERI, D FIGUEROA, J IRIARTE, N LEFEVRE, G PIZARRO, R QUIÑONES, M SOBARZO \& A TRONCOSO (1998) Producción primaria y su destino en la trama trófica pelágica y océano profundo e intercambio océano-atmósfera de $\mathrm{CO}_{2}$ en la zona norte de la corriente de Humboldt $\left(23^{\circ} \mathrm{S}\right)$ : posibles efectos del evento $\mathrm{El}$ Niño, 1997-98 en Chile. Revista Chilena de Historia Natural 71: 429-458.

GONZÁLEZ HE, V ORTIZ \& M SOBARZO (2000) The role of faecal material in the particulate organic carbon flux in the northern Humboldt Current, Chile $\left(23^{\circ} \mathrm{S}\right)$, before and during the 1997-1998 El Niño. Journal of Plankton Research 22: 499-529.

GONZÁLEZ HE, R GIESECKE, C VARGAS, M PAVEZ, J IRIARTE, P SANTIBÁÑEZ, L CASTRO, R ESCRIBANO \& F PAGÉS (2004) Carbon cycling through the pelagic foodweb in the northern Humboldt Current off Chile $\left(23^{\circ} \mathrm{S}\right)$. Journal of Marine Science 61: 572-584.

GONZÁLEZ-DÁVILA H (1995) The role of phytoplankton cells on the control of heavy metals concentrations in seawater. Marine Chemistry 48: 215-236.

HAMMOND D, J McMANUS, W BERENSON, CH MEREDITH, G KLINKHAMMER \& K COALE (2000) Diagenetic fractionation of $\mathrm{Ge}$ and $\mathrm{Si}$ in reducing sediments: The missing Ge sink and a possible mechanism to cause glacial/interglacial variations in oceanic $\mathrm{Ge} / \mathrm{Si}$. Geochimica et Cosmochimica Acta 64: 2453-2465. 
HELZ G, C MILLER, J CHARNOCK, J MOSSELMANS, R PATRICK, C GARNER \& D VAUGHAN (1996) Mechanism of molybdenum removal from the sea and its concentration in black shales: EXAFS evidence. Geochimica et Cosmochimica Acta 60: 3631-3642.

HOLM-HANSEN O, C LORENZEN, R HOLMES \& J STRICKLAND (1965) Fluorometric determination of chlorophyll. Journal of Ocean Exploration 30: 3-15.

IRIARTE J, G PIZARRO, $\mathrm{V}$ TRONCOSO \& $\mathrm{M}$ SOBARZO (2000) Primary production and biomass of size-fractioned phytoplankton of Antofagasta, Chile $\left(23-24^{\circ} \mathrm{S}\right)$ during pre-El Niño and El Niño 1997. Journal of Marine Systems 26: 37-51.

IRIARTE J \& H GONZÁLEZ (2004) Phytoplankton size structure during and after the 1997/1998 El Niño in coastal upwelling area of the northern Humboldt Current System. Marine Ecology Progress Series 269: 83-90.

JACOBS L \& S EMERSON (1982) Trace metal solubility in an anoxic fjord. Earth and Planetary Science Letter 60: 237-252

KLINKHAMMER G \& M BENDER (1980) The distribution of manganese in the Pacific Ocean. Earth and Planetary Science Letter 46: 361-384.

KREMLING K (1983) Determination of trace metals. En: Grasshoff K, M Ehrhardt \& K Kremling (eds) Methods of seawater analysis: 189-191. Verlah Chemie Press, Weinheim, Germany.

KREMLING K (1985) The distribution of cadmium, copper, nickel, manganese, and aluminum in surface waters of the open Atlantic and European shelf area. Deep-Sea Research 32: 531-555.

LE GALL A, D HYDES, P STATHAM, N MORLEY \& C HUNT (1999) Processes influencing distributions and concentrations of $\mathrm{Cd}, \mathrm{Cu}, \mathrm{Mn}$ and $\mathrm{Ni}$ at the northwest European shelf break. Marine Chemistry 68: $97-115$

LIBES S (1992) An introduction to marine biogeochemistry. John Wiley \& Sons Press, New, York, New York, USA. 532 pp.

LOSCHER B J DE JONG \& H DE BAAR (1998) The distribution and preferential biological uptake of cadmium at $6^{\circ} \mathrm{W}$ in the southern ocean. Marine Chemistry 62: 259-286.

MARÍN V, L RODRÍGUEZ, L VALLEJO, J FUENTESECA \& E OYARCE (1993) Efecto de la surgencia costera sobre la productividad primaria primaveral de bahía Mejillones del Sur (Antofagasta, Chile). Revista Chilena de Historia Natural 66: 479-491.

MARÍN V \& G OLIVARES (1999) Estacionalidad de la productividad primaria en bahía Mejillones del Sur (Chile): una aproximación proceso-funcional. Revista Chilena de Historia Natural 72: 629-641.

MARÍN V, R ESCRIBANO, L DELGADO, G OLIVARES \& P HIDALGO (2001) Nearshore circulation in a coastal upwelling site off the northern Humboldt current system. Continental Shelf Research 21: 1317-1329.

MARÍN V, L DELGADO \& R ESCRIBANO (2003) Upwelling shadows at Mejillones bay (northern Chilean coast): a remote sensing in situ analysis. Investigaciones Marinas (Chile) 31: 47-55.

MORFORD J \& S EMERSON (1999) The geochemistry of redox-sensitive trace metals in sediments. Geochimica et Cosmochimica Acta 63: 1735-1750.

MORFORD J, A RUSSELL \& S EMERSON (2001) Trace metal evidence for changes in the redox environment associated with the transition from terrigenous clay to diatomaceous sediment, Saanich Inlet, BC. Marine Geology 174: 355-369.

MULLER F, M TRANTER \& P BALLS (1994) Distribution and transport of chemical constituents in the Clyde estuary. Estuarine, Coastal and Shelf Science 39: 105-126.

MURRAY J, L CODISTOPI \& G FRIEDERICH (1995) Redox environments: the suboxic zone in the Black Sea. En: Huang C, C O’Melia \& J Morgan (eds) Aquatic chemistry: 157-176. American Chemical Society Press, Washington, District of Columbia, USA.

NAMEROFF T, L BALISTRIERI \& W MURRAY (2002) Suboxic trace metals geochemistry in the eastern tropical North Pacific. Geochimica et Cosmochimica Acta 66: 1139-1158.

NAVEA E \& O MIRANDA (1980) Ciclo anual de las condiciones oceanográficas en Mejillones del Sur. Revista de Biología Marina (Chile) 17: 97-133.

NOLTING R \& H DE BAAR (1994) Behavior of nickel, copper, zinc and cadmium in the upper $300 \mathrm{~m}$ of a transect in the southern ocean $\left(57^{\circ}-62^{\circ} \mathrm{S}, 49^{\circ} \mathrm{W}\right)$. Marine Chemistry 45: 225-242.

NORIKI S, N ISHIMORI, K HARADA \& S TSUNOGAI (1985) Removal of trace metals from seawater during a phytoplankton bloom as studied with sediments traps in Funka bay, Japan. Marine Chemistry 17: 75-89.

PARSONS T, Y MAITA \& C LALLI (1984) A manual of chemical and biological methods seawaters analysis. Pergamon Press, Oxford, United Kingdom. 173 pp.

PEMPKOWIAK J, J CHIFFOLEAU \& A STANISZEWSKI (2000) The vertical and horizontal distribution of selected trace metals in the Baltic Sea off Poland. Estuarine, Coastal and Shelf Science 51: 115-125.

POHL C G KATTNER \& M SCHULTZ-BALDES (1993) Cadmium, copper, lead and zinc on transect through Artic and eastern Atlantic surface and deep waters. Journal of Marine System 4: 17-29.

POHL C \& U HENNINGS (2005) The coupling of logterm trace metal terns to internal trace metal fluxes at the oxic-anoxic interface in the Gotland basin $\left(57^{\circ} 19,20^{\prime}\right.$ N; $20^{\circ} 03,00^{\prime}$ E), Baltic Sea. Journal of Marine System 56: 207-225.

PIZARRO G J IRIARTE V MONTECINOS (2002) Mesoscale primary production and bio-optical variability off Antofagasta $\left(23^{\circ}-24^{\circ} \mathrm{S}\right)$ during the transition to El Niño 1997-1998. Revista Chilena de Historia Natural 75: 201-215.

RODÍGUEZ L, P ZÁRATE \& YE OYARCE (1986) Producción primaria del fitoplancton y su relación con la temperatura, oxígeno, nutrientes y salinidad en la Bahía de Mejillones del Sur. Revista de Biología Marina (Chile) 22: 75-96.

RODRÍGUEZ L, V MARÍN, M FARÍAS \& E OYARCE (1991) Identification of an upwelling zone by remote sensing and in situ measurement. Mejillones del Sur Bay (Antofagasta- Chile). Scientia Marina 55: 467-473.

RODRÍGUEZ L \& R ESCRIBANO (1996) Bahía Antofagasta y bahía Mejillones del Sur: observaciones de la temperatura, penetración de la luz, biomasa y composición fitoplanctónica. Estudios Oceanológicos (Chile) 15: 75-85.

RUE E, G SMITH, G CUTTER \& K BRULAND (1997) The response of trace element redox couples to suboxic condition in the water column. Deep-Sea Research 44: 113-134. 
RUSSELL A \& J MORFORD (2001) The behavior of redox-sensitive metals across a laminates-massivelaminated transition in Saanich Inlet, British Columbia. Marine Geology 174: 341-354.

SAAGER P, H De BAAR \& R HOWLAND (1992) Cd, Zn, $\mathrm{Ni}$ and $\mathrm{Cu}$ in the Indian Ocean. Deep-Sea Research 39: 9-35.

SAÑUDO-WILHELMY $S, K$ OLSEN, J SCELFO, T FOSTER \& A FLEGAL (2002) Trace metal distributions off the Antartic Peninsula in the Weddell Sea. Marine Chemistry 77: 157-170.

SCHROPP S, F LEWIS, H WINDOM, J RYAN, F CALDER \& L BURNEY (1990) Interpretation of metal concentration in estuarine sediments of Florida using aluminum as a reference element. Estuaries 13: 227-235

SEGOVIA-ZAVALA J, F DELGADILLO-HINOJOSA \& S ÁLVAREZ-BORREGO (1998) Cadmium in the coastal upwelling area adjacent to the CaliforniaMexico border. Estuarine, Coastal and Shelf Science 46:475-781.

SOBARZO M \& D FIGUEROA (2001) The physical structure of a cold filament in a Chilean upwelling zone (Península de Mejillones, Chile, $23^{\circ} \mathrm{S}$ ). DeepSea Research 48: 2699-2726.

STEPHENS M, D KADKO, C SMITH \& M LATASA (1997) Chlorophyll-a pheopigments as tracers of labile organic carbon at the central equatorial Pacific seafloor. Geochimica et Cosmochimica Acta 61: 4605-4619.

STRUB P, J MESÍAS, V MONTECINOS, J RUTLLANT \& S MARCHANT (1998) Coastal oceanic circulation off western South America. En: Robinson A \& K Birnk (eds) The sea: 273-314. John Wiley, New York, New York, USA.

SUN M \& J DAI (2005) Relative influence of bioturbation and physical mixing on degradation of bloomderived particulate organic matter: clue from microcosm experiments. Marine Chemistry 96: 201218

TAKESUE R \& A VAN GEEN (2002) Nearshore circulation during upwelling inferred from the distribution of dissolved cadmium off the Oregon coast. Limnology and Oceanography 47: 176-185.

TAKESUE R, A VAN GEEN, J CARRIQUIRY, E ORTIZ, L GODINEZ-ORTA, I GRANADOS, M SALDÍVAR, L ORTLIEB, R ESCRIBANO, N GUZMÁN, J CASTILLA, M VARAS, M SALAMANCA \& C FIGUEROA (2004) Influence of coastal upwelling and El Niño-Southern Oscillation on nearshore water along Baja California and Chile: shore-based monitoring during 1997-2000. Journal of Geophysical Research 109: 1-14.

ULLOA O, R ESCRIBANO, S HORMAZÁBAL, R QUIÑÓNEZ, R GONZÁLEZ \& R RAMOS (2001) Evolution and biological effects of the 1997-98 El Niño in the upwelling ecosystem off northern Chile. Geophysical Research Letter 28: 1591-1594.

VALDÉS J (1998) Evolución oceanográfica reciente de la Bahía Mejillones del Sur $\left(23^{\circ} \mathrm{S}\right)$. Evidencia geoquímica en sedimentos marinos. Tesis doctoral, Centro EULA-Chile, Universidad de Concepción Concepción, Chile. 144 pp.

VALDÉS J, L LÓPEZ, S LOMÓNACO \& L ORTLIEB (2000) Condiciones paleoambientales de sedimentación y preservación de materia orgánica en Bahía Mejillones del Sur $\left(23^{\circ} \mathrm{S}\right)$, Chile. Revista de Biología Marina y Oceanografía (Chile) 35: 169 180.

VALDÉS J \& L ORTLIEB (2001) Paleoxigenación subsuperficial de la columna de agua en la bahía Mejillones del Sur $\left(23^{\circ} \mathrm{S}\right)$ : Indicadores geoquímicos en testigos de sedimento marino. Investigaciones Marinas (Chile) 29: 25-35.

VALDÉS J, L ORTLIEB \& A SIFEDDINE (2003) Variaciones del sistema de surgencia de Punta Angamos $\left(23^{\circ} \mathrm{S}\right)$ y la Zona de Mínimo Oxígeno durante el pasado reciente. Una aproximación desde el registro sedimentario de la bahía Mejillones del Sur. Revista Chilena de Historia Natural 76: 347 362.

VALDÉS J (2004) Evaluación de metales redox-sensitivos como proxies de paleoxigenación en un ambiente marino hipóxico del norte de Chile. Revista Chilena de Historia Natural 77: 121-138.

VALDÉS J, G VARGAS, A SIFEDDINE, L ORTLIEB \& M GUIÑEZ (2005) Distribution and enrichment evaluation of heavy metals in Mejillones Bay $\left(23^{\circ}\right.$ S), northern Chile: geochemical and statistica approach. Marine Pollution Bulletin 50: 1558-1568.

VAN GEEN A, S LUOMA, C FULLER, R ANIMA, E CLIFTON \& S TRUMBORE (1992) Evidence from $\mathrm{Cd} / \mathrm{Ca}$ ratio in foraminifera for greater upwelling off California 4,000 years ago. Nature 358: 54-56.

VAN GEEN A \& D HUSBY (1996) Cadmium in the California Current System: tracer of the past and present upwelling. Journal of Geophysical Research 101: 3489-3507.

VARGAS G (2002) Interactions ocean-atmosphère au cours des derniers siècles sur la côte du Désert d' Atacama: analyse multi-proxies des sédiments laminés de la Baie de Mejillones $\left(23^{\circ} \mathrm{S}\right)$. Tesis doctoral, Universidad de Bordeaux I, Francia. 270 pp.

VARGAS G, L ORTLIEB, J PICHON, J BERTAUX \& M PUJOS (2004) Sedimentary facies and high resolution primary production inferences from laminated datomacous sediments off northern Chile $\left(23^{\circ} \mathrm{S}\right)$. Marine Geology 211: 79-99.

VEH H (1967) Deposition of uranium from the ocean. Earth and Planetary Science Letter 3: 145-150.

YOON Y, JM MARTIN \& M COTTÉ (1999) Dissolved trace metals in the Western Mediterranean Sea total concentration and fraction isolated by $\mathrm{C} 18$ Sep-Pak technique. Marine Chemistry 66: 129-148.

ZHENG Y, R ANDERSON, A VAN GEEN \& J KUWABARA (2000) Authigenic molybdenum formation in marine sediments: a link to porewater sulfide in the Santa Barbara basin. Geochimica et Cosmochimica Acta 64: 4165-4178. 
